\title{
Singularity-theoretic methods in robot kinematics
}

\author{
P. S. Donelan \\ School of Mathematics, Statistics and Computer Science, \\ Victoria University of Wellington, \\ PO Box 600, Wellington, New Zealand \\ E-mail: peter.donelan@vuw.ac.nz
}

\begin{abstract}
The significance of singularities in the design and control of robot manipulators is well known and there is an extensive literature on the determination and analysis of singularities for a wide variety of serial and parallel manipulators - indeed such an analysis is an essential part of manipulator design. Singularity theory provides methodologies for a deeper analysis with the aim of classifying singularities, providing local models and local and global invariants. This paper surveys applications of singularity-theoretic methods in robot kinematics and presents some new results.
\end{abstract}

Keywords: Singularity theory; Robot manipulator; Transversality; Lie group; Genericity; Screw systems. 


\section{Introduction}

In simple terms, a singularity of a robot manipulator occurs where the number of instantaneous degrees of freedom of its end-effector, tool or platform differs from the expected number based on the degrees of freedom of its individual actuated joints, subject to constraints such as closed loops.

For a serial manipulator, the forward (direct) kinematics describes the position of the endeffector-parametrised in space by, say, $x_{1}, \ldots, x_{6}$ where three parameters correspond to translations, and three to rotations - as a function $f$ of the actuated joint variables $\theta_{1}, \ldots, \theta_{n}$. In any given configuration, that is for a particular choice of joint variables, the manipulator Jacobian is the linear relation between the joint velocities and the end-effector velocity (with respect to the parametrisation) given by the matrix of partial derivatives $\partial x_{i} / \partial \theta_{j}$. A singularity is any configuration for which the rank of the Jacobian drops below its maximum possible value, being the lesser of the number of joint variables $n$ and the dimension of the space of end-effector positions, which is 6 . This property is, of course, independent of the choice of parametrisation.

Examples of singular configurations for a serial manipulator with $n \leq 6$ revolute joints occur when:

- two joints in a concurrent 3-joint spherical wrist assembly are collinear, so that instantaneous rotation is only possible about axes in a plane through the wrist centre - a wrist singularity

- three joints are coplanar and parallel, so that the three joints instantaneously permit only rotation about one axis and translation perpendicular to the common plane - an elbow singularity.

Research on manipulator singularities dates back to the 1960s at least. Whitney ${ }^{1}$ identified singularities as an obstruction to certain control algorithms requiring the construction of inverse kinematic solutions. Singularities of the kind above were first described by Featherstone. ${ }^{2}$ Hunt $^{3}$ considered uncertainty configurations for single closed-loop mechanisms, where the mechanism has an instantaneous increase in mobility. Subsequently, Hunt et $a l^{4-6}$ linked the idea to singular configurations of robot arms by considering a virtual rigid connection between the end-effector and base. This idea was developed further by Litvin et $a l^{7,8}$ and Wang and Waldron. ${ }^{9}$

The situation for parallel manipulators is more complicated and was first clarified by Gosselin and Angeles. ${ }^{10}$ In principle, the kinematics may be described by an implicit function $F(\boldsymbol{\theta}, \mathbf{x})=0$, where the variables $\theta_{i}$ correspond to the actuated joints and the values of the function $F$ are $k^{-}$ dimensional. The Jacobian of $F$ partitions as $\left(\begin{array}{llll}J_{\boldsymbol{\theta}} & J_{\mathbf{x}}\end{array}\right)$. A drop in the rank of $J_{\boldsymbol{\theta}}$ has similar effect to that of a serial manipulator singularity: there is a loss of instantaneous degrees of freedom in the platform. A drop in the rank of $J_{\mathbf{x}}$ admits (instantaneous or sometimes finite) platform motion even though the actuated joints are stationary. An example of the first kind of singularity occurs when the lines of four of the actuated legs of a 3-3 Gough-Stewart platform intersect at one of the joints on the platform. The underlying Grassmann line geometry was explained by Merlet. ${ }^{11}$ The second kind, often denoted a constraint singularity, has been a subject of recent interest, for example for the 3 -UPU manipulator ${ }^{12,13}$ for which passive switching between translational and rotational modes is possible. Singularities of each type determine limits on the existence of forward and inverse kinematic functions for parallel manipulators.

An important related problem is to identify and analyze the trajectory singularities of points in the end-effector or platform. A nice example, which has received considerable attention, is the class of 3-dof regional manipulators. ${ }^{14-17}$ As was shown by Pieper and Roth, ${ }^{18}$ it is possible to 
solve the inverse kinematics for a wrist-partitioned 6-dof serial manipulator. The first three links determine a positioning sub-assembly (regional manipulator) while the last three form a spherical wrist sub-assembly. The Jacobian reflects this decomposition and partitions into $3 \times 3$ blocks as

$$
\left(\begin{array}{cc}
\mathbf{J}_{11} & \mathbf{J}_{12} \\
\mathbf{J}_{21} & \mathbf{O}
\end{array}\right)
$$

Thus, singularities of the whole mechanism are determined by singularities of the wrist subassembly ( $\operatorname{det} \mathbf{J}_{21}=0$ ) together with singularities of the position of the wrist centre $\left(\operatorname{det} \mathbf{J}_{12}=0\right.$ ). Since the wrist centre may be regarded as a design variable, it is important to understand the trajectory singularities of points of the end-effector of the regional manipulator. ${ }^{19}$

There are a number of fundamental reasons for analyzing singularities. Work- space boundary configurations (other than those arising from extremal values of joint variables) must be singular; more generally, if the number of postures of a manipulator is not constant for end-effector/platform positions near a given position, then that position corresponds to a singular configuration. Control mechanisms that rely on computation of an inverse or generalized inverse of a Jacobian are likely to breakdown near a singularity; associated to this may be unbounded joint accelerations and torques. Even when it is possible to avoid singularities, their presence can result in lack of repeatability. ${ }^{20,21}$ On the other hand, it is also the case that in singular configurations a manipulator may be able to withstand theoretically unboundedly large torques acting on its end-effector, leading to mechanical advantage. ${ }^{22}$

Singularity analyses of robot manipulators typically seek to identify singular configurations for a given manipulator architecture, usually dependent on a number of design parameters. Specifically, this may include: describing the geometric conditions for singularity, such as coincidence and collinearity conditions, as in the examples above; determining the geometry or topology of the singularity locus; and optimization with respect to design parameters, such as maximizing the singularity-free workspace or other singularity metrics.

Concepts and techniques from mathematical singularity theory help in answering these questions and at the same time prompt some deeper and more general questions about manipulator singularities.

1. Is it possible to classify the singularities of robot manipulators in terms of invariants of an appropriate equivalence relation? Are there associated normal forms or models for such a classification?

2. Are there certain singularity types that occur for a typical (generic) manipulator? How does this apply to a given class of manipulators? What can be said about non-generic manipulators, either in general or in a given class?

3. When is a manipulator qualitatively stable with respect to its singularity locus, $i e$ does the locus have the same topological structure under small perturbations of the design parameters?

4. How does the family of trajectory singularities relate to the underlying kinematics?

A number of researchers have explicitly used singularity-theoretic methods in contributions to the literature on manipulator singularities. The necessity for serial manipulators with general translational and rotational freedom to have singular configurations was first observed by Paul and Stevenson ${ }^{23}$ using Jacobians and proved in general by Gottlieb ${ }^{24}$ and Baker and Wampler ${ }^{25}$ using topological arguments. See Section 4. 
Tchon ${ }^{26,27}$ made the first explicit attempt to understand manipulator singularities in terms of the singularity theory of differentiable functions. In particular, he sought normal forms for singularities, but recognised that the special form of forward kinematic mappings for serial manipulators imposes significant restrictions on the applicability of so-called genericity results. The consequences were explored in a series of subsequent papers ${ }^{28-34}$ and is described in Section 5.3.

Pai and Leu ${ }^{35}$ independently derived theoretical conditions concerning the degree of singularity of serial manipulators. The goal was to establish generic properties of singular loci. They examined some specific wrist-partitioned architectures from this perspective. In particular, this involved calculating the second-order behaviour of the forward kinematics to characterise what are called transversality conditions. More general statements for serial manipulators, making explicit use of the product-of-exponentials formulation (see Section 3.2), were subsequently obtained by Hao and Lerbet ${ }^{36,37}$ and applied to the analysis of closed-loop mechanisms. It is worth noting that the mobility of a closed loop with $\leq 6$ joints is only possible along a set of singular configurations of the corresponding open chain. So the analysis of singular sets has special implications for understanding over-constrained mechanisms. Section 5.1 summarises this line of research.

Burdick $^{16}$ developed the distinction between generic and non-generic manipulators in a classification of $3 \mathrm{R}$ regional manipulators. Wenger et al ${ }^{17,38-40}$ have clarified the classification and pursued the consequences of this through the idea of cuspidal manipulators, where the presence of the non-repeatability phenomenon can be explained geometrically by the presence of cusp singularities. Although this work does not explicitly use singularity theory methods, the geometry revealed closely resembles classical results of that theory and suggests a basis for more explicit analysis. Other research that explores second-order behaviour to understand singularities is in the papers of Karger ${ }^{41-43}$ and Ghosal and Ravani. ${ }^{44}$ They also make explicit use of the Lie group structure that describes the general workspace.

The connection between a forward kinematic mapping and the singularities of trajectories of a rigid body has been a fundamental part of mechanism design since Watt. The author ${ }^{45}$ first analysed this in singularity-theoretic terms, while Gibson et al ${ }^{46-49}$ developed a methodology based on algebraic geometry for analysing specific classical mechanisms. This evolved into a project to classify kinematic singularities and to establish genericity theorems for specific classes of mechanism. ${ }^{50-57}$ The latter part of this project however faced the same difficulties identified by Tchon. A brief account of the basic ideas and some examples are discussed in Section 5.4.

The work of Marsh, Duffy et $a l^{58-60}$ should also be mentioned, though it is not dealt with further in this paper. It concerns parallel compliant mechanisms, where the use of springs gives rise to a potential function and static configurations correspond to local minima. This is the subject of a branch of singularity theory called catastrophe theory. This provides paradigms explaining 'catastrophic' or sudden changes in the configuration under the influence of small changes in external forces.

The aim of this paper is to present some of the mathematical concepts underlying singularity theory in the context of robot kinematics. For the most part, it is assumed that the reader is familiar with the terminology and concepts of robotics but not with the mathematical concepts. To that end, Sections 2 and 4 provide a necessarily brief presentation of the range of mathematical ideas and results required from differential topology and singularity theory. It has become increasingly clear that the application of these ideas in robotics needs to recognise the special form of kinematic mappings, relating to the Lie group structure of rigid body motions. Therefore Section 3 provides some of the necessary concepts concerning Lie groups, their Lie algebras, screws and screw systems. The author has attempted to ensure that the relevance of the mathematical concepts for robot kine- 
matics is maintained throughout by the inclusion of relevant examples. Section 5 brings together the preceding material in a summary of several applications of singularity-theoretic techniques in robot kinematics.

For greater detail, the mathematical foundations of robot kinematics that form an ideal background are in the books of Murray, Li and Sastry ${ }^{61}$ and Selig. ${ }^{62}$ For the underlying concepts of topological spaces see, for example, Munkres. ${ }^{63}$ The relevant language and results of differential topology appear in, for example, Guillemin and Pollack ${ }^{64}$ or Lee, ${ }^{65}$ the latter of which describes Lie groups from the point of view of differential topology. Mathematical details of the singularity theory of differentiable maps are presented in Gibson ${ }^{66}$ and, at a more technical level, Arnold et $a l,{ }^{67}$ Martinet $^{68}$ or Golubitsky and Guillemin. ${ }^{69}$

\section{Differentiable Manifolds and Mappings}

There are two main branches of singularity theory concerning, on the one hand, singularities of real and complex differentiable functions and, on the other, singularities of algebraic varieties and their morphisms. The two are closely connected and share a number of common ideas; both are informative about manipulator singularities, but the emphasis in this paper is on the former, which has attracted greater attention in the robotics literature. The language of this branch of singularity theory largely derives from differential topology and a brief summary, linked to its application in robotics is given here.

\subsection{Topological Spaces and Differentiable Manifolds}

The basic objects of study are differentiable manifolds and differentiable functions between them. Differentiable manifolds can be defined as special sorts of subsets of Euclidean spaces $\mathbb{R}^{N}$, though they do not always arise naturally in that way. A more fundamental approach is to view them as special kinds of topological space, the simplest setting in which one can define continuity. It is useful to adopt this approach here because other topological spaces will be introduced later that are not themselves differentiable manifolds. A topological space is simply a set that has a welldefined collection of open subsets; the open sets must include the whole space and the empty set, and finite intersections and arbitrary unions of open sets must be open. An open set containing a point $x$ is referred to as a neighbourhood of $x$. A closed set is one whose complement is open. Closed sets may be characterised by the property that they contain all their boundary points. The neighbourhoods, in effect, encode 'nearness', though in a less quantitative way than if there were a metric (distance function).

If $X, Y$ are topological spaces and $f: X \rightarrow Y$ a function, then for any subset $B \subset Y$, denote by $f^{-1}(B)$ the set $\{x \in X: f(x) \in B\}$ called the inverse image of $B$ under $f . f$ is continuous if and only if the inverse image of every open set in $Y$ is open in $X$. In the special case that $B$ contains a single point $y \in Y$, the notation for the inverse image is $f^{-1}(y)$ and this is variously called the fibre of $f$ over $y$ or the level set of $y$.

A basic example is the Euclidean space $\mathbb{R}^{n}$. Here, the Euclidean metric defines a topology. A simple open set is an open ball of radius $r>0$ centred at a point $\mathbf{c} \in \mathbb{R}^{n}$ :

$$
B(\mathbf{c}, r)=\left\{\mathbf{x} \in \mathbb{R}^{n}:\|\mathbf{x}-\mathbf{c}\|<r\right\} .
$$


By definition, a set $A \subseteq \mathbb{R}^{n}$ is open (in the Euclidean sense) if for every point $\mathbf{x} \in A$, there is some $r>0$ so that $B(\mathbf{x}, r) \subseteq A$. In particular, if $A$ is a non-empty open neighbourhood of a point $\mathbf{x}$, then it contains all sufficiently small perturbations of $\mathbf{x}$. That is a useful empirical characterisation of openness, even when there is no explicit notion of distance given.

A differentiable manifold, $M$, is a topological space that can be parametrised on some neighbourhood of every point $x \in M$ by Euclidean coordinates of a fixed dimension $n$, the dimension of the manifold. A parametrisation is a bijective function $\phi: U \rightarrow \phi(U) \subseteq M$ where $U \subseteq \mathbb{R}^{n}$ is a non-empty open subset and $\phi(U) \subseteq M$ is also open; further $\phi$ and its inverse $\phi^{-1}: \phi(\bar{U}) \rightarrow U$ should be continuous. The inverse is also called a coordinate chart. Differentiability is introduced by requiring that coordinate transformations between overlapping parametrisations be differentiable, usually taken to mean smooth or $C^{\infty}$, that is, partial derivatives of all orders exist and are continuous.

A non-trivial but simple example of a differentiable manifold is the unit circle $S^{1}=\{(x, y) \in$ $\left.\mathbb{R}^{2}: x^{2}+y^{2}=1\right\}$, which is a 1 -dimensional manifold. It can be parametrised by angle $\theta \mapsto$ $(\cos \theta, \sin \theta)^{t}$. Of course the angle does not have a unique value since $\theta+2 n \pi$ parametrises the same point in $S^{1}$ for all integers $n$, but the requirement that parametrisations be one-to-one means that two such values cannot lie in the domain of a single parametrisation. For example, the parametrisations $\phi_{1}:(-\pi, 0) \rightarrow S^{1}$ and $\phi_{2}:(\pi / 2,3 \pi / 2) \rightarrow S^{1}$ are distinct, but they overlap on $\phi_{1}(-\pi,-\pi / 2)=\phi_{2}(\pi, 3 \pi / 2) \subset S^{1}$. The coordinate transformation $\phi_{2}^{-1} \circ \phi_{1}:(-\pi,-\pi / 2) \rightarrow$ $(\pi, 3 \pi / 2)$ is the differentiable (in this case linear) function $\theta \mapsto \theta+2 \pi$. In practice, one does not usually need to be too concerned about the precise domains of parametrisation. Note that $S^{1}$ sits inside a Euclidean space $\mathbb{R}^{2}$ of higher dimension and $S^{1}$ is said to be a submanifold of $\mathbb{R}^{2}$.

To avoid pathological examples, a manifold should have enough open sets that any 2 distinct points can be separated by disjoint open neighbourhoods (the Hausdorff property) and some countable collection of open sets such that every open set can be formed as a union of some of them (second countability). Returning to the Euclidean space $\mathbb{R}^{n}$, this is trivially an $n$-dimensional manifold since it parametrises itself. Any two distinct points $\mathbf{c}_{1}, \mathbf{c}_{2}$ are separated by the disjoint open balls centred at each, whose radius is half (or less) the distance between them. An appropriate basic countable collection of open sets is the set of open balls with rational radii and rational coordinates for their centres. The Hausdorff property ensures that any finite set of points is closed. Any level set $f^{-1}(y) \subseteq M$ of a continuous function $f: M \rightarrow N$ between manifolds is a closed set.

A subset $S$ of a topological space $X$ is dense if there is at least one point of $S$ in every open subset of $X$. For example, the points with rational coordinates form a dense subset of $\mathbb{R}^{2}$. If $S \subset X$ is dense then every point $x \in X$ is near $S$, in the sense that every open neighbourhood of $x$ contains some $s \in S$.

The immediate relevance of Section 2.1 for robot kinematics is that manipulator jointspaces and workspaces, as well as sets of design parameters, are differentiable manifolds. Singularity theory places particular emphasis on properties of topological spaces that hold on open and dense sets since these may therefore be regarded as stable (unaffected by small perturbation) and ubiquitous.

\subsection{Jointspaces and Workspaces}

A revolute ( $R$ ) joint requires the coincidence of two circles, one in each of the joined links, so its jointspace is the set of orientation- and angle-preserving (rigid) transformations from a circle to itself. Such a transformation can be represented by a linear transformation of the plane containing 
the circles, ie a $2 \times 2$ matrix $A$, that preserves inner or dot product (and hence angle and length) and also orientation:

1. for all $\mathbf{u}, \mathbf{v} \in \mathbb{R}^{2}, A \mathbf{u} . A \mathbf{v}=\mathbf{u} . \mathbf{v}$, or equivalently $A A^{t}=I_{2}$

2. $\operatorname{det} A=1$.

The set of such matrices is the special orthogonal group, denoted $S O(2)$. The term group here means that

- the identity transformation is in $S O(2)$

- there is a well-defined associative composition of transformations, given by matrix multiplication

- each element in $S O(2)$ has a well-defined inverse.

For any $A \in S O(2)$, the conditions 1. and 2. entail that for some $\alpha \in \mathbb{R}$ :

$$
A=\left(\begin{array}{cc}
\cos \alpha & -\sin \alpha \\
\sin \alpha & \cos \alpha
\end{array}\right)
$$

It is clear, as a result, that $S O(2)$ is effectively the same as $S^{1}$ since each is parametrised by angles - they are said to be diffeomorphic manifolds.

A useful shorthand for the representation (2) is to write $A=e^{\alpha J}$, where $J=\left(\begin{array}{cc}0 & -1 \\ 1 & 0\end{array}\right)$. This form for $A$ can be deduced by comparison of the entries of the matrix series

$$
e^{B}=\sum_{n=0}^{\infty} \frac{B^{n}}{n !}
$$

using the substitution $B=\alpha J$, and the Taylor series for $\sin$ and $\cos$ in (2). It is also a straightforward computation that

$$
e^{\alpha J} e^{\beta J}=e^{(\alpha+\beta) J} .
$$

It follows that in $S O(2)$ composition commutes or is independent of order. A group with that property is called abelian. The property is, in any case, clear from geometric intuition about composition of planar rotations.

For a prismatic $(\mathrm{P})$ joint, representing displacement along the axis relative to a home configuration, and a helical $(\mathrm{H})$ joint, representing rotation about an axis at a constant rate relative to a non-zero displacement along it, the jointspace is (diffeomorphic to) the 1-dimensional manifold $\mathbb{R}$ (the real number line). The jointspace for a cylindrical (C) joint is the cylinder $S^{1} \times \mathbb{R}$. Products of manifolds are also manifolds whose dimension is the sum of the dimensions of its components, therefore two in this case.

For a spherical (S) joint, the jointspace, disregarding constraints imposed by the construction of the joint, is the 3-dimensional manifold $S O(3)$ consisting of orientation-preserving and distancepreserving transformations of a (unit) sphere $S^{2}=\left\{(x, y, z) \in \mathbb{R}^{3} \mid x^{2}+y^{2}+z^{2}=1\right\}$ to itself. This represents the required coincidence between spherical joint components on adjacent links. There are several well known ways of parametrising $S O(3)$, for example using Euler angles, but note that, 
as for $S O(2)$, no parametrisation suffices for the whole manifold, at least if one wishes to ensure that the domain of parametrisation is open and the parameter values unique. Unlike $S O(2)$, the group $S O(3)$ is not abelian.

For a serial manipulator, the combined jointspace is the Cartesian product of the jointspace for each joint so that, if the manipulator consists of 6 revolute joints, the jointspace is $S^{1} \times \ldots \times S^{1}$ (6 copies) and has dimension 6. Such a product of circles is known as a (generalised) torus. The 2-dimensional torus $S^{1} \times S^{1}$ can be pictured as the surface of a tyre inner-tube or a bagel.

The workspace of a manipulator is the set of positions of its end-effector or platform or, sometimes, as mentioned in Section 1, the position of a specified point of the end-effector. The general position, relative to some chosen home position, is defined by means of a Euclidean transformation of affine $n$-space ( $n=2$ for a planar manipulator, $n=3$ for a spatial manipulator), which is a map preserving orientation and distance. Given a choice of origin and coordinate system identifying affine $n$-space with $\mathbb{R}^{n}$, such a transformation can be decomposed as a rotation $A \in S O(n)$ about the origin followed by a translation $\mathbf{a} \in \mathbb{R}^{n}$, so that

$$
\mathbf{x} \mapsto A \mathbf{x}+\mathbf{a} .
$$

The set of such transformations $(A, \mathbf{a})$ is denoted $S E(n)$ and it is diffeomorphic as a manifold to the product $S O(n) \times \mathbb{R}^{n}$. Note that composition of transformations does not simply work component-wise in this product; in fact:

$$
\left(A_{1}, \mathbf{a}_{1}\right) \cdot\left(A_{2}, \mathbf{a}_{2}\right)=\left(A_{1} A_{2}, A_{1} \mathbf{a}_{2}+\mathbf{a}_{1}\right) .
$$

In group theory, this is referred to as a semi-direct product.

The function described by equation (5) is called an action of the group $S E(n)$ on the manifold $\mathbb{R}^{n}$. In general, the action of a group $G$, with identity element $e$, on a manifold $M$ is a function $G \times M \rightarrow M,(g, x) \mapsto g \cdot x$, such that $e \cdot x=x$ for all $x \in M$ and $\left(g_{2} . g_{1}\right) \cdot x=g_{2} \cdot\left(g_{1} \cdot x\right)$ for all $g_{1}, g_{2} \in G$ and $x \in M$. Indeed, this is really the point of a group - that it represents a collection of symmetries of some object, in this case a Euclidean rigid body. Note, however, that having abstracted the underlying group from its 'natural' action, it may well have interesting actions on other manifolds.

Group actions are fundamental in classification schemes. An action of a group $G$ on a manifold $M$ partitions $M$ into equivalence classes or orbits:

$$
G \cdot x=\{y \in M: y=g \cdot x \text { for some } g \in G\} .
$$

The orbits, or sometimes unions of orbits, may be regarded as natural classes of objects in $M$ with respect to the action of $G$.

\subsection{Differentiable Functions, Derivatives and Singularities}

Given differentiable manifolds $M$ and $N$ of dimensions $m$ and $n$ respectively, a function $f: M \rightarrow N$ is differentiable if for any point $x \in M$ there are parametrisations $\phi$ of a neighbourhood of $x$ and $\psi$ of a neighbourhood of $y=f(x) \in N$ so that the local representative of $f, \psi^{-1} \circ f \circ \phi$, is a differentiable function between open subsets of $\mathbb{R}^{m}$ and $\mathbb{R}^{n}$. For example, if $A=e^{\alpha J} \in S O(2)$ for fixed $\alpha$, then there is a differentiable function $\rho_{\alpha}: S^{1} \rightarrow S^{1}$ given by $\mathbf{x} \mapsto A \mathbf{x}$, the natural action of $S O(2)$ on $S^{1}$. If $\mathbf{x}=(\cos \theta, \sin \theta)^{t}$, then using angular parametrisations, it can be readily checked that a local representative of $f$ is given by $\theta \mapsto \theta+\alpha$. 
While $M$ and $N$ may not themselves be linear spaces, the $n \times m$ Jacobian matrix of partial derivatives of a function representing $f$ defines a linear map $\mathbb{R}^{m} \rightarrow \mathbb{R}^{n}$. The matrix itself is obviously dependent on the choice of parametrisations for $M$ and $N$. In fact, one can associate with each point $x \in M$ an $m$-dimensional vector space called the tangent space of $M$ at $x$, denoted $T_{x} M$. Elements of $T_{x} M$ are called tangent vectors. There are several equivalent ways to do this for abstract manifolds though if $M$ is a submanifold of a Euclidean space $\mathbb{R}^{N}$ then the tangent space can be identified in a natural way with the geometric idea of space tangent to a multidimensional surface. In any case, there is a basis for $T_{x} M$ associated to any given parametrisation of a neighbourhood of $x$, and likewise for $y \in N$, such that the Jacobian of the local representative of $f$ is the matrix representation of a linear map, denoted $T_{x} f$, called the tangent map or derivative of $f$ at $x$, between the tangent spaces $T_{x} M$ and $T_{f(x)} N$. This derivative satisfies the Chain Rule: if $f: M \rightarrow N$ and $g: N \rightarrow P$ then $T_{x}(g \circ f)=T_{f}(x) g \cdot T_{x} f$. The right-hand side can be evaluated as the product of representative Jacobian matrices.

Definition 2.1. A singularity or critical point of a differentiable function $f: M \rightarrow N$ is a point $x \in M$ at which the derivative $T_{x} f$ has rank less than $\min \{\operatorname{dim} M, \operatorname{dim} N\}$. The corank of a singularity $x \in M$ is the difference $\min \{\operatorname{dim} M, \operatorname{dim} N\}-\operatorname{rank} T_{x} f$. The point $x$ is called regular if $T_{x} f$ has full rank. A point $y \in N$ is called a regular value if every point $x \in f^{-1}(y)$ is a regular point.

The corank provides a basic stratification of singularities by increasing degeneracy.

The following result is a simple paradigm for more powerful results of singularity theory:

Theorem 2.2. Let $M, N$ be differentiable manifolds of dimensions $m, n$ and $f: M \rightarrow N$ be a differentiable function. If $y \in N$ is a regular value and the level set $P=f^{-1}(y)$ is non-empty then $P$ is a submanifold of $M$ of dimension $m-n$. Moreover the tangent space $T_{x} P$ is the kernel of the derivative $T_{x} f$.

The difference between the dimensions of a submanifold $P$ and the manifold $M$ it sits in is called the codimension of $P$ in $M$, denoted $\operatorname{codim} P$. Thinking of a single point $\{y\} \subset N$ as a 0 -dimensional submanifold, the theorem states that for $y$ regular, the codimensions of $\{y\}$ and $P$ are equal.

The special orthogonal group $S O(n)$ consists of $n \times n$ real matrices $A$ that preserve the Euclidean inner product on $\mathbb{R}^{n}$, hence $A A^{t}=I_{n}$, and have $\operatorname{det} A=1$. They can be constructed as submanifolds using the theorem, as one component of the inverse image of the identity matrix in $S(n)$, the manifold of symmetric $n \times n$ matrices, under the function $f: M(n) \rightarrow S(n), f(A)=A A^{t}$. Since $\operatorname{dim} S(n)=\frac{1}{2} n(n+1)$, we have $\operatorname{dim} S O(n)=n-\frac{1}{2} n(n+1)=\frac{1}{2} n(n-1)$. The derivative of $f$ is $T_{A} f(B)=A B^{t}+B A$ so, in particular, the tangent space at the identity $A=I$ consists of the matrices satisfying $B^{t}+B=0$, ie skew-symmetric matrices.

Given a planar or spatial serial manipulator with jointspace $\Theta$ and workspace $S E(n)(n=2$ or 3 ), the kinematic mapping (forward kinematics) is a differentiable function $f: \Theta \rightarrow S E(n)$. Given a kinematic mapping $f$ with jointspace $\Theta$, suppose $x \in \Theta$ is a configuration of the manipulator. A tangent vector $v \in T_{x} \Theta$ is a joint velocity vector. If, as above, there is a parametrisation $\phi$ of the jointspace $\Theta$ in terms of joint angles $\theta_{1}, \ldots, \theta_{k}$ then $v$ is represented by the vector $\left(\dot{\theta}_{1}, \ldots, \dot{\theta}_{k}\right)$. The tangent map $T_{x} f$ transforms joint velocity vectors $v$ into workspace velocity vectors that lie in a tangent space to the workspace $S E(3)$, described in Section 3.

Example. Consider a 3R planar serial manipulator whose joints, in a given home configuration, have position vectors $\mathbf{a}_{i}$ with respect to a coordinate system in the base and joint variables $\theta_{i}$, 
$i=1,2,3$. Let $\mathbf{d}_{i}=\mathbf{a}_{i}-\mathbf{a}_{i-1}$ for $i=2,3$, then the kinematic mapping is given by the composition

$$
\begin{aligned}
f\left(\theta_{1}, \theta_{2}, \theta_{3}\right) & =\left(e^{\theta_{1} J}, \mathbf{a}_{1}\right) \cdot\left(e^{\theta_{2} J}, \mathbf{d}_{2}\right) \cdot\left(e^{\theta_{3} J}, \mathbf{d}_{3}\right) \\
& =\left(e^{\theta_{1} J} e^{\theta_{2} J} e^{\theta_{3} J}, e^{\theta_{1} J} e^{\theta_{2} J} \mathbf{d}_{3}+e^{\theta_{1} J} \mathbf{d}_{2}+\mathbf{a}_{1}\right)
\end{aligned}
$$

Strictly, this should be thought of as $\chi^{-1} \circ f \circ \phi$, where $\phi$ is a parametrisation of the jointspace by the real numbers $\theta_{1}, \theta_{2}, \theta_{3}$, each in some interval such as $(-\pi, \pi)$, and $\chi$ is the identification of $S E(2)$ with the product $S O(2) \times \mathbb{R}^{2}$ arising from choices of coordinates in the base and end-

effector. As observed above (2), $S O(2)$ can be parametrised by angle $\alpha$. Then, applying (4), a local representative of $f$ is

$$
\left(\theta_{1}, \theta_{2}, \theta_{3}\right) \mapsto\left(\theta_{1}+\theta_{2}+\theta_{3}, e^{\theta_{1} J} e^{\theta_{2} J} \mathbf{d}_{3}+e^{\theta_{1} J} \mathbf{d}_{2}+\mathbf{a}_{1}\right) .
$$

Using the fact that $J$ is the matrix corresponding to a rotation by $\pi / 2$, the derivative of this map at a general point $\left(\theta_{1}, \theta_{2}, \theta_{3}\right)$ is represented by the Jacobian matrix of partial derivatives which, writing its bottom two rows as a row of 2 -vectors, has the form:

$$
\left(\begin{array}{ccc}
1 & 1 & 1 \\
e^{\left(\theta_{1}+\theta_{2}+\pi / 2\right) J} \mathbf{d}_{3}+e^{\left(\theta_{1}+\pi / 2\right) J} \mathbf{d}_{2} & e^{\left(\theta_{1}+\theta_{2}+\pi / 2\right) J} \mathbf{d}_{3} & \mathbf{0}
\end{array}\right)
$$

This fails to have rank 3 , and hence has a singularity, when $e^{\left(\theta_{1}+\theta_{2}+\pi / 2\right) J} \mathbf{d}_{3}$ and $e^{\left(\theta_{1}+\pi / 2\right) J} \mathbf{d}_{2}$ are proportional, that is when the three joints $\mathbf{a}_{1}, \mathbf{a}_{2}, \mathbf{a}_{3}$ are collinear. In such a configuration, instantaneous translation of the end-effector in the direction of the line of the joints is impossible.

A second key theorem concerning differentiable functions says that their regular values are always plentiful.

Theorem 2.3 (Morse-Sard-Brown Theorem). For any differentiable function $f: M \rightarrow N$ between differentiable manifolds, the set of regular values is dense in $N$.

In fact there is a stronger statement, that the critical values form a set of measure zero, but that requires introducing a further technical definition. Note that the theorem says nothing about the set of regular points in $M$.

It follows from Theorems 2.2 and 2.3 that, given a kinematic mapping $f: \Theta \rightarrow S E(3)$ for a manipulator with $\geq 6$ joints, for almost all positions of the end-effector in $p \in S E(3)$, the selfmotion set $f^{-1}(p)$ is either empty or a manifold whose dimension is the degree of redundancy $\operatorname{dim} \Theta-6 .{ }^{16}$

\subsection{Transversality}

Central to many of the results and applications of singularity theory is the notion of transversality, which can be regarded as a generalisation of regularity. The idea is to encode a property of elements of a manifold $M$ by requiring the value of some differentiable function $f: M \rightarrow N$ on the manifold to lie in a submanifold $Q$ of $N$. For example, suppose $M$ is the jointspace of a manipulator and $f: M \rightarrow S E(3)$ its forward kinematic mapping. The set of configurations of the manipulator for which the end-effector has a fixed orientation $R$ is defined by the property $f\left(\theta_{1}, \ldots, \theta_{k}\right) \in Q$ where given a choice of coordinates identifying $S E(3)$ with $S O(3) \times \mathbb{R}^{3}, Q=\{(A, \mathbf{a}): A=R\}$. One then seeks a condition that ensures the subset of $M$ characterised by this property is itself well behaved, for example also a submanifold. 
Definition 2.4. Let $M$ and $N$ be differentiable manifolds of dimensions $m, n$ respectively and $Q \subset N$ a submanifold with $\operatorname{dim} Q=q$. A differentiable function $f: M \rightarrow N$ is transverse to $Q$ if for all $x \in f^{-1}(Q)$, the inverse image of $Q$,

$$
T_{f(x)} Q+T_{x} f\left(T_{x} M\right)=T_{f(x)} N .
$$

Transversality is denoted symbolically by $f$ 历 $Q$. The definition means that at each $x \in f^{-1}(Q)$, the set of sums of vectors in the image of the derivative of $f$ at $x$ and in the tangent space to $Q$ at $f(x)$ span the whole tangent space to $N$ at $f(x)$. Checking for transversality is, in principle, just a question of linear algebra-one must check that a set of $(q+m) n$-vectors spans $n$-space. Writing the vectors as columns of a matrix, this is equivalent to requiring some $n \times n$ submatrix to have non-zero determinant. Of course, this has to be established generally for all points in the inverse image, which may require the use of more than one local representative of $f$. However, for serial manipulators with revolute joints a single formula for $f$ in terms of joint angles is likely to suffice. Clearly a necessary condition for (10) to hold is that $q+m \geq n$. Put another way, if $q+m<n$ and $f \pitchfork Q$ then $f^{-1}(Q)$ is empty. For the case where $Q$ consists of a single point $y \in N$, so $q=0$, transversality to $Q$ is equivalent to requiring that $y$ is a regular value, since the derivative at any $x \in f^{-1}(Q)$ must be onto and hence of rank $n$. So regularity is indeed a special case of transversality.

The following result generalises Theorem 2.2.

Theorem 2.5. If $f: M \rightarrow N$ is differentiable and transverse to a submanifold $Q \subset N$, then, so long as it is non-empty, $P=f^{-1}(Q) \subset M$ is a submanifold and $\operatorname{codim} P=\operatorname{codim} Q$.

There is also an extension of the Morse-Sard-Brown Theorem 2.3. The theorem concerns a family of functions, parametrised by some manifold $B$. This fits well with robot kinematics, since one often deals with such families where $B$ represents the set of design parameters for a class of manipulators.

Theorem 2.6 (Elementary Transversality Theorem). Let $M, N$ and $B$ be differentiable manifolds and $Q$ a submanifold of $N$. Let $F: M \times B \rightarrow N$ be a differentiable function and for each $b \in B$ denote by $F_{b}: M \rightarrow N$ the function $F_{b}(x)=F(x, b)$. If $F \pitchfork Q$ then for a dense set of parameters $b \in B, F_{b} \pitchfork Q$.

Note that it is much easier for $F$ to meet the transversality condition since its domain has dimension $\operatorname{dim} M+\operatorname{dim} B$, whereas $F_{b}$ has domain of dimension $\operatorname{dim} M$ only. Hence, the bigger the number of parameters, the more likely it is that the hypothesis of the theorem may hold.

\section{The Euclidean Group and its Lie Algebra}

\subsection{Lie Groups and Lie Algebras}

The jointspaces $G$ of the individual joints and the workspace $S E(n)$ are simultaneously manifolds and transformation groups. Moreover, the operations of composition and inverse define differentiable functions on $G$. Such an object is called a Lie group. The tangent space $T_{e} G$ at the identity element $e$ of a Lie group $G$ represents the set of infinitesimal transformations corresponding to the transformations embodied in an action of the group. It has a special binary operation $[X, Y]$ called 
the Lie bracket whose value is the infinitesimal transformation corresponding to the difference between the compositions of $X$ and $Y$ in that, and in the reverse, order. This makes $T_{e} G$ into a Lie algebra, usually denoted $\mathfrak{g}$. The Lie bracket is bilinear (linear in each factor), antisymmetric, $[X, Y]=-[Y, X]$, and satisfies the Jacobi identity:

$$
[X,[Y, Z]]+[Y,[Z, X]]+[Z,[X, Y]]=0 .
$$

The Lie groups of interest in robotics can be regarded as matrix groups: submanifolds of the $n^{2}$-dimensional vector space and manifold $M(n)$ of $n \times n$ matrices. Note that $M(n)$ is diffeomorphic to $\mathbb{R}^{n^{2}}$. In this case the tangent space can also be constructed as a set of $n \times n$ matrices. For matrix groups, the Lie bracket is the commutator:

$$
\left[B_{1}, B_{2}\right]=B_{1} B_{2}-B_{2} B_{1}
$$

which vanishes if and only if the matrices commute. So, for example, the Lie bracket for $\mathfrak{s o}(2)$ is identically zero, since $S O(2)$ is abelian.

For the special orthogonal group $S O(n)$, as we saw in Section 2.3, the Lie algebra is the set $\mathfrak{s o}(n)$ of skew-symmetric matrices. In the case $n=2, \operatorname{dim} \mathfrak{s o}(2)=1$ and the Lie algebra is spanned by the matrix $J$ introduced in Section 2.1. For $n=3$, $\operatorname{dim} \mathfrak{s o}(3)=3$, and there is a canonical isomorphism between 3 -vectors $\boldsymbol{\omega}$ and elements of the Lie algebra $\hat{\boldsymbol{\omega}}$ :

$$
\boldsymbol{\omega}=\left(\begin{array}{l}
\omega_{1} \\
\omega_{2} \\
\omega_{3}
\end{array}\right) \longleftrightarrow \hat{\boldsymbol{\omega}}=\left(\begin{array}{ccc}
0 & -\omega_{3} & \omega_{2} \\
\omega_{3} & 0 & -\omega_{1} \\
-\omega_{2} & \omega_{1} & 0
\end{array}\right)
$$

Note that if $\boldsymbol{\omega} \neq \mathbf{0}$, it spans the kernel of $\hat{\boldsymbol{\omega}}$, i.e. $\hat{\boldsymbol{\omega}}(\boldsymbol{\omega})=\mathbf{0}$.

The elements of $S E(3)$, as described above, are not matrices; given a choice of coordinates, they form a pair consisting of an orthogonal matrix $A$ and a 3 -vector a. However the group has a familiar 4-dimensional representation in which $(A, \mathbf{a})$ is identified with the partition matrix $\left(\begin{array}{cc}A & \mathbf{a} \\ 0 & 1\end{array}\right)$ and the action on $\mathbf{x} \in \mathbb{R}^{3}$ is recovered by embedding $\mathbb{R}^{3}$ as $\left(\begin{array}{c}\mathbf{x} \\ 1\end{array}\right) \in \mathbb{R}^{4}$.

Elements of the Lie algebra $\mathfrak{s e}(3)$ are called twists: infinitesimal transformations of 3-space. They can also be represented by $4 \times 4$ matrices of the form

$$
\left(\begin{array}{cc}
\hat{\omega} & \mathbf{v} \\
0 & 0
\end{array}\right)
$$

This also provides for representation by the 6 -vector $\boldsymbol{\xi}=\left(\omega_{1}, \omega_{2}, \omega_{3}, v_{1}, v_{2}, v_{3}\right)^{t}$ and the matrix form (11) is then denoted $\hat{\boldsymbol{\xi}}$. The coordinates $\omega_{i}, v_{i}$ are frequently referred to as the Plücker coordinates of the twist, by extension from classical line geometry. Applying the commutator to the matrix form (11) one can deduce that in Plücker coordinates the Lie bracket is given by

$$
\left[\boldsymbol{\xi}_{1}, \boldsymbol{\xi}_{2}\right]=\left(\begin{array}{c}
\boldsymbol{\omega}_{1} \wedge \boldsymbol{\omega}_{2} \\
\boldsymbol{\omega}_{1} \wedge \mathbf{v}_{2}-\boldsymbol{\omega}_{2} \wedge \mathbf{v}_{1}
\end{array}\right)
$$

where $\wedge$ represents the standard vector product in $\mathbb{R}^{3}$.

These representations are dependent on specific choices of base and end-effector coordinates. A simultaneous change of coordinates that fixes the identity is a conjugation in $S O(3) \times \mathbb{R}^{3}$, that is $g \mapsto$ 
$h g h^{-1}$, where $h$ represents the coordinate change. Differentiating with respect to $g$ and evaluating at $g=e$ gives a corresponding action of the Lie group on its Lie algebra $\operatorname{Ad}(h) \cdot X=h X h^{-1}$, called the adjoint action. Here, the right-hand side can be calculated by matrix multiplication using the representations above. In terms of Plücker coordinates, it has the form: ${ }^{62}$

$$
\operatorname{Ad}(A, \mathbf{a}) \cdot\left(\begin{array}{l}
\boldsymbol{\omega} \\
\mathbf{v}
\end{array}\right)=\left(\begin{array}{c}
A \boldsymbol{\omega} \\
A \mathbf{v}-(A \boldsymbol{\omega}) \wedge \mathbf{a}
\end{array}\right)
$$

Associated with this (linear) action of the Euclidean group is a natural extension to an action on polynomial functions of the Plücker coordinates: $g \cdot f(\boldsymbol{\omega}, \mathbf{v})=f(g \cdot(\boldsymbol{\omega}, \mathbf{v}))$. There are two fundamental quadratic polynomials that are invariant under this action (that is, there value is constant on orbits of the action): the Killing form $\boldsymbol{\omega} \cdot \boldsymbol{\omega}$ and the Klein form $\boldsymbol{\omega} \cdot \mathbf{v}$. These play a central role in the classification of screws and screw systems in Section 3.3.

A final important observation is that the adjoint action itself is a function whose variable lies in the group. Its derivative, the adjoint action of the Lie algebra, is precisely the Lie bracket:

$$
\operatorname{ad}\left(\boldsymbol{\xi}_{1}\right) \cdot \boldsymbol{\xi}_{2}=\left[\boldsymbol{\xi}_{1}, \boldsymbol{\xi}_{2}\right]
$$

\subsection{The Exponential Function}

The representation of elements of $S O(2)$ in the exponential form $e^{\alpha J}$ can be generalised to an arbitrary Lie group $G$. Given a fixed element $g$ of a Lie group $G$ there are two differentiable functions $G \rightarrow G$ given by right and left composition with $g: R_{g}(h)=h g, L_{g}(h)=g h$. These give rise to the derivatives $T_{e} R_{g}, T_{e} L_{g}: \mathfrak{g} \rightarrow T_{g} G$ which are bijective linear functions (isomorphisms) between the tangent spaces at the identity and at any point $g \in G$. For matrix groups, where $e$ is the identity matrix $I$, if $g=A \in G$ and $B \in \mathfrak{g}$, then simply

$$
T_{I} R_{A}(B)=B A, \quad T_{I} L_{A}(B)=A B
$$

Conversely the inverses of these derivatives act as right and left pullbacks of the tangent space at any $h \in G$ into the Lie algebra - in the matrix case, simply $B \mapsto B A^{-1}, B \mapsto A^{-1} B$ respectively. Composing the pullbacks with a kinematic mapping $f: \Theta \rightarrow S E(3)$ provides two ways of forming a Jacobian representing a linear map from the joint velocity space $T_{\theta} \Theta$ into the Lie algebra, representing position velocities. In this setting, left (resp. right) pullback correspond to making a change of coordinates in the manipulator base (resp. end-effector) in such a way that at the given configuration, the kinematic mapping is the identity.

Given an element $v$ in the Lie algebra $\mathfrak{g}$, left translation can be used to define a corresponding tangent vector $v(g) \in T_{g} G$ for all $g \in G$. The collection of tangent vectors is called a left-invariant vector field. There is a $1-1$ correspondence between left-invariant vector fields and elements of $\mathfrak{g}$. The vector field is, in effect, a system of differential equations on $G$ and integration gives rise to curves $p_{v}: \mathbb{R} \rightarrow G$ with the property that $\dot{p}_{v}(t)=v\left(p_{v}(t)\right)$ for all $t \in \mathbb{R}$. Integral curves with initial condition $p_{v}(0)=e$ (the identity in $G$ ) satisfy the property $p_{v}\left(t_{1}+t_{2}\right)=p_{v}\left(t_{1}\right) \cdot p_{v}\left(t_{2}\right)$ for any $t_{1}, t_{2} \in \mathbb{R}$, where the right-hand side is a composition in $G$. Hence, the set $\left\{p_{v}(t): t \in \mathbb{R}\right\}$ is a subgroup of $G$, called a one-parameter subgroup.

The exponential function on the Lie algebra $\mathfrak{g}$ is the map $\exp : \mathfrak{g} \rightarrow G$ defined by $\exp (v)=p_{v}(1)$ where $p_{v}$ is the integral curve with $p_{v}(0)=e$. For the multiplicative group of positive real numbers, $\mathbb{R}^{+}$, the Lie algebra is $\mathbb{R}$ and this function is precisely the familiar exponential function $\exp (x)=e^{x}$. 
It is frequently useful to adopt this index notation for a general Lie group. In fact, the exponential function parametrises the group $G$ on some neighbourhood of the identity. The coordinates for $G$ so derived from a given basis for $\mathfrak{g}$ are called exponential coordinates of the first kind. In general, exp may be neither injective (one-to-one) nor surjective (onto) and it may be necessary therefore to restrict exp to an open subset of $\mathbf{0} \in \mathfrak{g}$. It is in fact surjective for $S E(3)$.

For any matrix Lie group, the exponential function is given by the (necessarily convergent) series (3). For any $X \in \mathfrak{g}$, the set $\{\exp (t X): t \in \mathbb{R}\}$ is a one-dimensional Lie subgroup, called a one-parameter subgroup of $G$. In particular, $(\exp (t X))^{-1}=\exp (-t X)$. Differentiating the series gives

$$
\frac{d}{d t}(\exp t X)=X \exp t X
$$

In particular, at $t=0$ the derivative is simply $X$. The group property means that, analogous to $(4), e^{t_{1} X} \cdot e^{t_{2} X}=e^{\left(t_{1}+t_{2}\right) X}$. However it is not the case in general that $e^{X} \cdot e^{Y}=e^{X+Y}$, unless $[X, Y]=0$.

Theorem 3.1 (Campbell-Baker-Hausdorff). If $G$ is a Lie group with Lie algebra $\mathfrak{g}$, Lie bracket $[\cdot, \cdot]$ and $X, Y \in \mathfrak{g}$, then for $X$ and $Y$ sufficiently small, $e^{X} e^{Y}=e^{X * Y}$, where $X * Y$ is a convergent series beginning

$$
\begin{aligned}
X+Y+\frac{1}{2}[X, Y]+\frac{1}{12}([ & X,[X, Y]]-[Y,[X, Y]]) \\
& -\frac{1}{48}([X,[Y,[X, Y]]]+[Y,[X,[X, Y]]])+\cdots
\end{aligned}
$$

The exponential function and the Campbell-Baker-Hausdorff formula are of fundamental importance in manipulator kinematics. The relative motion between links connected by the 1-dof lower pairs correspond precisely to the 1-parameter subgroups of $S E(3)$. The motions generated by any such joint can therefore be expressed in the form $e^{\theta X}$ for some twist $X \in \mathfrak{s e}(3)$.

For a general serial manipulator with $k 1$-dof joints, the kinematic mapping can be written as a product of exponentials in $S E(3)$, as first observed by Brockett: ${ }^{71}$

$$
f\left(\theta_{1}, \theta_{2}, \ldots, \theta_{k}\right)=H \cdot e^{\theta_{1} X_{1}} \cdot e^{\theta_{2} X_{2}} \cdots e^{\theta_{k} X_{k}}
$$

Here, $H \in S E(3)$ is a constant Euclidean transformation that simply determines the home configuration $\left(\theta_{1}=\cdots=\theta_{k}=0\right)$ of the end-effector with respect to the base - that is, it identifies the moving coordinates with the ambient coordinates in the home configuration. One may choose coordinates so that $H$ is the identity. $X_{1}, \ldots X_{k}$ are the twists of each joint in respect of the motion of the end-effector with respect to the base, regarding all the other joints as fixed. Another form of writing this is to choose a coordinate frame in each link in such a way that the relative motion of link $L_{i}$ to link $L_{i-1}$ is described by a twist in some standard form (say, rotation about the $z$-axis in the $i$ the frame). That then requires interpolation of matrices between each exponential corresponding to the coordinate transformation between consecutive links in the home position. These matrices can be encoded in terms of the well-known Denavit-Hartenberg parameters. ${ }^{72}$

Setting $H=I$, and using (15), differentiating with respect to $\theta_{i}$ and setting the joint variables to 0 simply gives the twist $X_{i}$. Therefore the twists $X_{1}, \ldots, X_{k}$ describe the infinitesimal capabilities of the manipulator in the home configuration. Another way to think of this is that the twists, written in Plücker coordinates, form the columns of the Jacobian. 
The Campbell-Baker-Hausdorff formula (16) applied to the kinematic mapping (17), with $H=I$, gives

$$
f\left(\theta_{1}, \ldots, \theta_{k}\right)=\exp \left(\sum_{i=1}^{k} \theta_{i} X_{i}+\frac{1}{2} \sum_{1 \leq i<j \leq k} \theta_{i} \theta_{j}\left[X_{i}, X_{j}\right]+O(3)\right)
$$

where the order 3 terms (in the $\theta_{i} \mathrm{~s}$ ) involve higher order brackets of the $X_{i} \mathrm{~s}$. It follows that the workspace is contained in the exponential image of the Lie subalgebra generated by the twists $X_{1}, \ldots, X_{k}$. There is a classification of the subalgebras of $S E(3)$ : they are in one-to-one correspondence with the Lie subgroups classified by Hervé. ${ }^{73}$ Simple examples are the 3 -dimensional subalgebras consisting of infinitesimal rotations about a point or of infinitesimal translations. It is worth noting that there are no 5-dimensional subalgebras so that the subalgebra generated by any set of 5 linearly independent twists has to be the whole algebra $\mathfrak{s e}(3)$.

Where one is interested in a manipulator architecture possessing a number of design parameters, it is preferable, mathematically at least, to maintain a fixed jointspace. For serial manipulators with 1-dof joints it is worth distinguishing several general classes of architecture:

1. $\mathcal{R}$ : manipulator architectures with $m \mathrm{R}$-joints for all choices of design parameters. In this case the jointspace can be taken as $\left(S^{1}\right)^{m}$.

2. $\mathcal{R} \mathcal{P}$ : manipulator architectures with a combination of $\ell \mathrm{R}$-joints and $m-\ell \mathrm{P}$-joints which have a given order for all choices of design parameters. The jointspace is $\left(S^{1}\right)^{\ell} \times \mathbb{R}^{m-\ell}$. (Strictly, this is a union of classes over all possible orders of $\mathrm{R}$ and $\mathrm{P}$.)

3. $\mathcal{O}$ : (open) manipulator architectures with $m$ arbitrary $(\mathrm{R}, \mathrm{P}$ or $\mathrm{H})$ joints, which may vary with the design parameters. The jointspace is taken to be $\mathbb{R}^{m}$.

\subsection{Screws and Screw Systems}

A non-zero scalar multiple of the twist $X$ representing a given joint gives the same relative motion (one-parameter subgroup), so that it is only the screw corresponding to $X$, that is the set of its non-zero multiples, that uniquely determines a joint. Hence, in geometric terms, screws are simply elements of the projective space of lines through the origin in $\mathfrak{s e}(3)$.

To classify screws, the adjoint action of the Euclidean group defines an equivalence relation and classes may be taken to be the orbits or natural unions of orbits. Normal forms can be found for the different types of joint via the adjoint action (13). The pitch of a twist or its corresponding screw is the ratio of the fundamental invariants introduced at the end of Section $3.1 h=\boldsymbol{\omega} \cdot \mathbf{v} / \boldsymbol{\omega} \cdot \boldsymbol{\omega}$, so long as $\boldsymbol{\omega} \neq \mathbf{0}$; for $\boldsymbol{\omega}=\mathbf{0}$ set $h=\infty$. For $h \neq \infty$, it measures the signed displacement along the axis resulting from one complete rotation about the axis and the pitch separates orbits. The classification and its relation to the standard joint types is as follows:

\begin{tabular}{lccc}
\hline Joint type & Label & Normal form & Pitch \\
\hline revolute & $\mathrm{R}$ & $(1,0,0,0,0,0)^{t}$ & 0 \\
helical & $\mathrm{H}$ & $(1,0,0, h, 0,0)^{t}$ & $h$ \\
prismatic & $\mathrm{P}$ & $(0,0,0,1,0,0)^{t}$ & $\infty$ \\
\hline
\end{tabular}


Denote by $S \subseteq \mathfrak{s e}(3)$ the subspace spanned by $X_{1}, \ldots, X_{k}$. This is called a screw system, abbreviated to $k$-system if the subspace has dimension $k$. Clearly screw systems have maximum dimension 6 , so for a redundant manipulator, where $k>6$, one does not obtain a $k$-system. Similarly, if the manipulator has a singularity at its home configuration, then the associated screw system has dimension $<k$.

Screw systems of a given dimension $k$ form a manifold, known as a Grassmannian manifold, which has dimension $k(6-k)$. Since the adjoint action (13) is linear, it transforms screw systems to screw systems and can therefore be used to define a natural equivalence on $k$-systems. A classification was originally proposed by $\mathrm{Hunt}^{3}$ and this was given a mathematical foundation by Gibson and Hunt. ${ }^{74,75}$ Gibson and Donelan ${ }^{76}$ proved that the classes form a natural stratification of the Grassmannian into submanifolds.

The basis of the classification is in terms of the intersection of the screw system with the family of orbits of the adjoint action on the Lie algebra, parametrised by pitch, and in particular the class of screws of infinite pitch. Type I systems do not lie wholly in an orbit of fixed pitch while type II systems do. Type I systems may be further distinguished by the family of intersections, which is a pencil of conics. The subtypes A, B, C, D, for each type, distinguish the (projective) dimension of intersection with the orbit of pitch $\infty$ : subtype A denoting empty intersection, up to subtype D denoting a 2-dimensional intersection. Table 1 lists the classification, giving the type, a basis of screws in normal form is given and the codimension of the class as a submanifold of the Grassmannian.

\begin{tabular}{|l|l|c|c|l|c|}
\hline type & basis & codim & type & basis & codim \\
\hline \hline $\mathrm{IA}_{1}$ & $\left(1,0,0 ; h_{\alpha}, 0,0\right)$ & 0 & IIA & $(1,0,0 ; h, 0,0)$ & 5 \\
& $\left(0,1,0 ; 0, h_{\beta}, 0\right)$ & & & $(0,1,0 ; 0, h, 0)$ & \\
& $\left(0,0,1 ; 0,0, h_{\gamma}\right)$ & & & $(0,0,1 ; 0,0, h)$ & \\
\hline $\mathrm{IA}_{2}$ & $\left(1,0,0 ; h_{\alpha}, 0,0\right)$ & 2 & IIB & $(1,0,0 ; h, 0,0)$ & 5 \\
& $\left(0,1,0 ; 0, h_{\beta}, 0\right)$ & & & $(0,1,0 ; 0, h, 0)$ & \\
& $\left(0,0,1 ; 0,0, h_{\beta}\right)$ & & & $(0,0,0 ; 0,0,1)$ & \\
\hline $\mathrm{IB}_{0}$ & $(1,0,0 ; h, 0,0)$ & 1 & IIC & $(1,0,0 ; h, 0,0)$ & 6 \\
& $(0,1,0 ; 0, h, 0)$ & & & $(0,0,0 ; 0,1,0)$ & \\
& $(0,0,0 ; 1,0, p)$ & & & $(0,0,0 ; 0,0,1)$ & \\
\hline IB $_{3}$ & $\left(1,0,0 ; h_{\alpha}, 0,0\right)$ & 3 & IID & $(0,0,0 ; 1,0,0)$ & 9 \\
& $\left(0,1,0 ; 0, h_{\beta}, 0\right)$ & & & $(0,0,0 ; 0,1,0)$ & \\
& $(0,0,0 ; 0,0,1)$ & & & $(0,0,0 ; 0,0,1)$ & \\
\hline $\mathrm{IC}^{2}$ & $(1,0,0 ; 0,0,0)$ & 4 & & & \\
& $(0,0,0 ; 0,1,0)$ & & & & \\
& $(0,0,0 ; 1,0, p)$ & & & & \\
\hline
\end{tabular}

Table 1: Classification of 3-systems.

For $k>3$, the classification relies on reciprocity of screw systems. Associated to the Klein form 
(Section 3.1) is a bilinear form $B_{0}$ on $\mathfrak{s e}(3) .{ }^{74}$ Associated with any $k$-system $S$ is a $(6-k)$-system

$$
S^{\perp}=\left\{(\boldsymbol{\omega}, \mathbf{v}) \in \mathfrak{s e}(3): \forall\left(\boldsymbol{\omega}_{1}, \mathbf{v}_{1}\right) \in S, B_{0}\left((\boldsymbol{\omega}, \mathbf{v}),\left(\boldsymbol{\omega}_{1}, \mathbf{v}_{1}\right)\right)=0\right\} .
$$

The classification of $(6-k)$-systems induces a classification of $k$-systems. Note that the classification of 3 -systems is self-reciprocal.

Given a kinematic mapping $f: \Theta \rightarrow S E(3)$, where $\operatorname{dim} \Theta=k$, suppose that $\theta \in \Theta$ is a regular configuration and $f(\theta)=e$, the identity in $S E(3)$. Then $T_{\theta} f\left(T_{\theta} \Theta\right) \subseteq \mathfrak{s e}(3)$ is a $k$-system. The pullback construction described in Section 3.2 enables one to associate a screw system with each configuration $\theta \in \Theta$, though its dimension will be $<k$ at a singular configuration.

If the kinematic mapping of a serial manipulator is the product of exponentials (17), then clearly the screw system at $\theta_{1}=\cdots=\theta_{k}=0$ is that spanned by $X_{1}, \ldots, X_{k}$. However, for $\theta_{i}=t_{i}$, $i=1, \ldots, k$ say, the associated screw system is likely to vary. To express the kinematic mapping in such a way that the displacement corresponding to the new parameter values is the identity in $S E(3)$ requires a change of coordinates. In the case $k=2$, set $\eta_{i}=\theta_{i}-t_{i}, i=1,2$ :

$$
\begin{aligned}
& e^{\theta_{1} X_{1}} e^{\theta_{2} X_{2}} e^{-t_{2} X_{2}} e^{-t_{1} X_{1}} \\
= & e^{\left(\theta_{1}-t_{1}\right) X_{1}} e^{t_{1} X_{1}} e^{\left(\theta_{2}-t_{2}\right) X_{2}} e^{-t_{1} X_{1}} \\
= & e^{\eta_{1} X_{1}} e^{t_{1} X_{1}} e^{\eta_{2} X_{2}} e^{-t_{1} X_{1}} .
\end{aligned}
$$

The corresponding twists $X_{i}\left(t_{1}, t_{2}\right)$ are found by differentiating with respect to $\eta_{i}$ and setting $\eta_{i}=0, i=1,2$. From the definition of the adjoint action and (15), this gives $X_{1}\left(t_{1}, t_{2}\right)=X_{1}$ and $X_{2}\left(t_{1}, t_{2}\right)=\operatorname{Ad}\left(e^{t_{1} X_{1}}\right) X_{2}$. By extension to general $k$, the twists $Y_{j}$ spanning the screw system at $\theta_{i}=t_{i}, i=1, \ldots, k$ are given by:

$$
Y_{j}=X_{j}\left(t_{1}, \ldots, t_{k}\right)=\operatorname{Ad}\left(e^{t_{1} X_{1}} \cdots e^{t_{j-1} X_{j-1}}\right) X_{j}, \quad 1 \leq j \leq k .
$$

One may be interested simply in the screw system spanned by the $Y_{j}$ s or more explicitly in the ordered set $\left(Y_{1}, \ldots, Y_{k}\right)$, which can be identified with the corresponding Jacobian matrix whose columns are the $Y_{j} \mathrm{~s}$.

One consequence of this is that while the product-of-exponentials formula (17) defines the kinematic mapping of a serial manipulator, it is only relative to a choice of home position. Any two sets of $k$ twists related by (20) define the same manipulator. A mathematical classification of serial manipulators would use (20) and global changes of body and ambient coordinates to define an equivalence relation on ordered sets of twists.

\section{Singularity Theory}

Singularity theory of differentiable functions between manifolds, say $M$ and $N$, aims to understand singularities in the context of the set of all such functions, denoted $C^{\infty}(M, N)$. Since this requires the domain $M$ and the range $N$ to be fixed, it is preferable to work within classes of manipulator that share the same jointspace and workspace, though this requirement can be weakened if the jointspaces collectively form a higher-dimensional manifold. There are global and a local aspects to the theory. Locally, one seeks a classification and normal forms for singularities. Globally, one aims to understand how the presence and types of singularities are related or constrained by the overall topology of the manifolds $M$ and $N$. 
The following theorem, global in character, due to Gottlieb, ${ }^{24}$ is fundamental for robot kinematics in that it asserts that there is no serial manipulator architecture, capable of full spatial freedom, that is singularity-free.

Theorem 4.1. For any serial manipulator, with jointspace $\Theta=T^{p} \times \mathbb{R}^{6-p}$, the kinematic mapping $f: \Theta \rightarrow S E(3)$ possesses singularities. Moreover there is no continuous function $g: S E(3) \rightarrow \Theta$ such that $g(f(\theta))=\theta$.

The obstruction to the existence of such a function lies in the topology of the rotational component of $S E(3)$. Similarly, an extension of the theorem due to Baker and Wampler ${ }^{25}$ and, independently, Gottlieb ${ }^{77}$ shows that even 'pointing' robots (where rotations in $S O(3)$ are replaced by directions in $S^{2}$ ) cannot be singularity-free. The theorem also generalises to say that for redundant manipulators, there is no global continuous inverse kinematic mapping. The proofs use techniques of algebraic topology that are outside the main concerns of this article (see, for example, Munkres ${ }^{63}$ ).

In this section, the essential language and concepts of singularity theory are set out. Technical details and a wide range of theorems can be found in, for example, Arnold et al, ${ }^{67}$ Golubitsky and Guillemin $^{69}$ or Martinet. ${ }^{68}$ The key steps are to define:

- a notion of equivalence ( $\mathcal{A}$-equivalence) of functions (globally or locally) to provide the basis for a classification;

- a topology (Whitney $C^{\infty}$ topology) on the set of functions as a basis for describing stability and genericity;

- a tool for establishing genericity (Thom Transversality Theorem).

\subsection{Equivalence of Functions}

A natural equivalence relation for differentiable manifolds is diffeomorphism - a differentiable function with differentiable inverse. Diffeomorphisms preserve all topological properties, such as dimension. An example was given in Section 2.2 of the circle $S^{1}$ and planar rotation group $S O(2)$. Diffeomorphisms give rise to a natural equivalence relation for differentiable functions between manifolds as follows. Suppose $f_{1}, f_{2} \in C^{\infty}(M, N)$. If there are diffeomorphisms $g, h$ of $M, N$, respectively, so that the following diagram commutes:

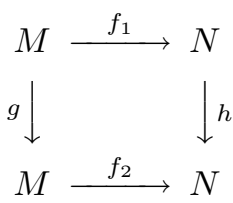

that is $f_{2}=h \circ f_{1} \circ g^{-1}$ then $f_{1}, f_{2}$ are said to be right-left or $\mathcal{A}$-equivalent. (The diffeomorphisms compose on the right and left of the function. In fact, the sets of diffeomorphisms on $M$ and $N$ form groups so this can also be regarded as an equivalence under a group action.) There are certainly other possible equivalences, for example simply right or left equivalence, where $h, g$ respectively are taken to be the identity function, but their application is more restricted.

A local version of $\mathcal{A}$-equivalence for functions $f_{i}$ at $x_{i} \in M, i=1,2$, is to require that there exist local representatives $f_{i} \circ \phi_{i}, i=1,2$ that are $\mathcal{A}$-equivalent on some neighbourhoods of $\phi_{i}^{-1}\left(x_{i}\right)$, 
$i=1,2$. In the commuting diagram (21), $M, N$ can be replaced by $\mathbb{R}^{m}, \mathbb{R}^{n}$ respectively and there is no loss of generality in assuming $x_{i}=\mathbf{0} \in \mathbb{R}^{m}, f_{i}\left(x_{i}\right)=\mathbf{0} \in \mathbb{R}^{n}$. Since the neighbourhood of $\mathbf{0}$ on which equivalence holds is arbitrary, the formal approach adopted in singularity theory is to consider germs of functions; that is, equivalence classes of all functions that are pairwise equal on some neighbourhood of $\mathbf{0}$ (however small). Denote the set of germs of functions $\left(\mathbb{R}^{m}, \mathbf{0}\right) \rightarrow\left(\mathbb{R}^{n}, \mathbf{0}\right)$ by $C^{\infty}\left(\mathbb{R}^{m}, \mathbb{R}^{n}\right)_{(0,0)}$. The set of germs has a nice algebraic structure (it is a local ring) which can be exploited in analysing local singularities but that is not pursued in this presentation. A basic problem is to classify singularities under local $\mathcal{A}$-equivalence and to find normal forms for each singularity type.

For a diffeomorphism, the derivative at every point has maximum rank, ie the common dimension of its domain and range. Suppose $x_{1} \in M$ and $x_{2}=g\left(x_{1}\right) \in N$, then applying the Chain Rule,

$$
T_{x_{2}} f_{2}=T_{x_{2}}\left(h \circ f_{1} \circ g^{-1}\right)=T_{f_{1}\left(x_{1}\right)} h \cdot T_{x_{1}} f_{1} \cdot T_{x_{2}} g^{-1}
$$

and hence the rank of $f_{2}$ at $x_{2}$ is the same as that of $f_{1}$ at $x_{1}$ by a basic result of linear algebra; in particular if $f_{1}$ has a singularity at $x_{1}$ then so does $f_{2}$ at $x_{2}$ and the corank (Definition 2.1) is a singularity invariant. On the other hand, $f_{1}$ and $f_{2}$ may have singularities at $x_{1}, x_{2}$ respectively, indeed may be of the same corank, and yet not be locally equivalent. Hence, finding invariants or normal forms that distinguish between inequivalent singularities is a major goal. Furthermore, one requires recognition principles, frequently based on invariants, to determine to which normal form a given singularity is equivalent.

\subsection{Jets and Jet Bundles}

In order to make descriptive statements about $C^{\infty}(M, N)$, it is valuable to give it the structure of a topological space. This should take account of the value of the function and all its partial derivatives of all orders. A useful way to keep track of this information is to consider the Taylor series expansion of representatives of a function $f \in C^{\infty}(M, N)$ at each point of $M$ and, in fact, the sequence of Taylor polynomials (truncated series).

It is easier to describe the construction precisely in the special case $M=\mathbb{R}^{m}, N=\mathbb{R}^{n}$. Suppose $f: \mathbb{R}^{m} \rightarrow \mathbb{R}^{n}, x_{0} \in \mathbb{R}^{m}$ and $k \geq 0$ is an integer. The $k$-jet of $f$ at $x_{0}, j^{k} f\left(x_{0}\right)$, is the pair $\left(x_{0}, T^{k} f\left(x_{0}\right)\right)$, where $T^{k} f\left(x_{0}\right)$ is the Taylor series expansion of $f$ at $x_{0}$, truncated beyond $k$-th order terms. The $k$-jet of $f$ (sometimes called the $k$-jet extension) is the function which assigns to each $x \in M$ the $k$-jet at $x: x \mapsto j^{k} f(x)$. Clearly this can be written as an $n$-vector of degree $k$ polynomials in $m$ variables. Note that $j^{0} f\left(x_{0}\right)=\left(x_{0}, f\left(x_{0}\right)\right) \in \mathbb{R}^{m} \times \mathbb{R}^{n}$. For $k \geq 0$, the $k$-jet $j^{k} f\left(x_{0}\right)$ lies in a vector space $J^{k}\left(\mathbb{R}^{m}, \mathbb{R}^{n}\right)$, called a $k$-jet bundle. The term 'bundle' arises from the idea that the space can be regarded as a collection of vector spaces

$$
\mathbb{R}^{m} \times \mathbb{R}^{n} \times J^{k}\left(\mathbb{R}^{m}, \mathbb{R}^{n}\right)_{(0,0)}
$$

where the last factor, the fibre of the bundle, consists of $n$-tuples of Taylor polynomials at $\mathbf{0} \in \mathbb{R}^{m}$ with zero constant term. The component in $\mathbb{R}^{m}$ is referred to as the source of the jet, and that in $\mathbb{R}^{n}$ as the target.

The general case, where $M, N$ are differentiable manifolds, can be dealt with by using local representatives of functions $M \rightarrow N$. The technical definition can be found in Golubitsky and Guillemin. ${ }^{69}$ Then $J^{k}(M, N)$ retains a bundle structure (technically it is a vector bundle over the base $M \times N$ ) and it looks locally like (22). It is itself a differentiable manifold, and for $f: M \rightarrow N$ 
the jet map $j^{k} f: M \rightarrow J^{k}(M, N)$ is a differentiable function. In the case $k=1$, only the linear terms of the Taylor series expansion are required and the fibre at $(x, y) \in M \times N$ can be thought of as the set $L\left(T_{x} M, T_{y} N\right)$ of linear maps between the relevant tangent spaces.

For example, expanding the Taylor series for $\sin \left(\theta+\theta_{0}\right)$ about $\theta=0$ and truncating gives

$$
\sin \theta_{0}+\cos \theta_{0} \cdot \theta-\frac{1}{2} \sin \theta_{0} \cdot \theta^{2} .
$$

The relevant information is contained in the coefficients with respect to $\theta$ so, keeping track of the source and target, one can write

$$
j^{2} \sin (x)=\left(x, \sin x, \cos x,-\frac{1}{2} \sin x\right) .
$$

Applying this expansion to the local representation $\hat{f}$ in equation (8), expanding the Taylor series about $\theta_{1}=x, \theta_{2}=y, \theta_{3}=z$ gives the 1 -jet:

$$
\begin{aligned}
j^{1} \hat{f}(x, y, z)= & \left(\begin{array}{l}
x \\
y \\
z
\end{array}\right),\left(\begin{array}{c}
x+y+z \\
1 \\
1 \\
1
\end{array}\right), \\
& \left(\begin{array}{c}
d_{31} \cos (x+y)-d_{32} \sin (x+y)+d_{21} \cos x-d_{22} \sin x+a_{11} \\
-d_{31} \sin (x+y)-d_{32} \cos (x+y)-d_{21} \sin x-d_{22} \cos x \\
-d_{31} \sin (x+y)-d_{32} \cos (x+y) \\
0
\end{array}\right), \\
& \left.\left(\begin{array}{c}
d_{31} \sin (x+y)+d_{32} \cos (x+y)+d_{21} \sin x+d_{22} \cos x+a_{12} \\
d_{31} \cos (x+y)-d_{32} \sin (x+y)+d_{21} \cos x-d_{22} \sin x \\
d_{31} \cos (x+y)-d_{32} \sin (x+y) \\
0
\end{array}\right)\right)
\end{aligned}
$$

where, as usual, the 3-vector at the beginning simply identifies the source of the jet, and the three 4 -vectors consist of the coefficients of the first-order Taylor series of the three components of $\hat{f}$. In the 2-jet, each component would contribute a further three terms corresponding to the coefficients of quadratic terms in the Taylor series.

Corank $r$ singularities. The value of the jet bundles is that it is frequently possible to characterise features of functions (or germs), especially those relating to singularities, by submanifolds of the jet bundle. The simplest case, $k=1$, is that there are well-defined submanifolds $\Sigma^{r} \subseteq J^{1}(M, N), r \geq 0$, so that for $f \in C^{\infty}(M, N)$ and $x \in M, j^{1} f(x) \in \Sigma^{r}$ if and only if $f$ has a singularity of corank $r$ at $x$. $\Sigma^{0}$ corresponds to non-singular jets and forms an open subset of the jet bundle. As indicated above, these submanifolds are certainly $\mathcal{A}$-invariant but, except for $\Sigma^{0}$, they are not 1-determined; in fact for any 1-jet in $\Sigma^{r}, r \geq 1$ there are infinitely many possible $\mathcal{A}$-equivalence classes with that 1 -jet. For example, in the case, $M=N=\mathbb{R}$, each function $f(x)=x^{k}, k \geq 2$, determines a distinct singular class (of corank 1 ) at $x=0$.

Returning to the general case, given manifolds $M, N$ of dimensions $m, n$ respectively, the set

$$
\Sigma^{r}=\left\{\sigma \in J^{1}(M, N): \operatorname{corank} T_{x} f=r \text { where } j^{1} f(x)=\sigma\right\}
$$

is a submanifold of codimension $r(|m-n|+r)$. Given $f: M \rightarrow N$, define $\Sigma^{r} f=\left(j^{1} f\right)^{-1}\left(\Sigma^{r}\right)$. This is the set of singular points of $f$ of corank $r$ and if $j^{1} f \pi \Sigma^{r}$, it is a submanifold of $M$ by the 
Transversality Pre-Image Theorem. Clearly the relative values of $m, n$ and $r$ constrain when this can happen non-trivially: they must satisfy

$$
m \geq r(|m-n|+r)
$$

The precise condition for transversality at some point $x \in \Sigma^{r} f$ is that the image of the derivative of the 1-jet extension should span a complement to the tangent space to $\Sigma^{r}$ at $j^{1} f(x)$. The following Lemma ${ }^{69}$ provides a direct means of determining transversaility.

Lemma 4.2. A necessary and sufficient condition for $j^{1} f \pi \Sigma^{r}$ is that the intrinsic second derivative, obtained by restricting the derivative of the 1-jet extension to the kernel of $T_{x} f$ and projecting it onto the quotient of $T_{f(x)} N$ by the image of $T_{x} f$, be surjective.

In simpler matrix terms, suppose that one is able to choose coordinates on $M$ and $N$ so that the matrix representative of $T_{x} f$ is the partitioned matrix $\left(\begin{array}{ll}\mathbf{I}_{q} & \mathbf{0}_{q \times(n-q)} \\ \mathbf{0}_{(m-q) \times q} & \mathbf{0}_{(m-q) \times(n-q)}\end{array}\right)$ where $q=\operatorname{rank} T_{x} f=\min \{m, n\}-r$. Then one requires that the $m$ derivatives of $j^{1} f$ at $x$, with respect to the coordinates in $M$, be a set of $m$ matrices whose bottom right-hand corners, consisting of $(m-q) \times(n-q)$ submatrices, span the vector space of all matrices of those dimensions. Implications for robot kinematics are considered in Section 5.1.

A further extension of the jet bundle concept enables global theorems about singularities to be established. The $r$-fold $k$-jet bundle $J_{r}^{k}(M, N)$ consists of an $r$-tuple of $k$-jets with $r$ distinct sources $x_{1}, \ldots, x_{r}$. Associated to a function $f: M \rightarrow N$ and $r$ distinct points $x_{1}, \ldots, x_{r}$ is a multijet

$$
j_{r}^{k} f\left(x_{1}, \ldots, x_{r}\right)=f\left(j^{k} f\left(x_{1}\right), \ldots, j^{k} f\left(x_{r}\right)\right) .
$$

\subsection{Stability and Genericity}

The jet bundles $J^{k}(M, N), k \rightarrow \infty$, act as a sequence of finite-dimensional approximations to $C^{\infty}(M, N)$. Each jet bundle has a natural topology derived from those of $M, N$ and the vector space of polynomials and these can be used to define a topology for $C^{\infty}(M, N)$ as follows. For any open set $U \in J^{k}(M, N)$, define

$$
\hat{U}=\left\{f \in C^{\infty}(M, N): j^{k} f(x) \in U \text { for all } x \in M\right\} .
$$

The union of all such $\hat{U}$, for all $k \geq 0$ and all open $U$, is the basis for a topology on $C^{\infty}(M, N)$, called the Whitney $C^{\infty}$ topology. A typical neighbourhood of a function $f \in C^{\infty}(M, N)$ is a set of functions $g$ such that for every $x \in M$, the value of each partial derivative of (a local representative of) $g$ at $x$ lies in an open interval about the corresponding partial derivative of $f$. The intervals may vary continuously across $M$; if $M$ is compact, i.e. homeomorphic to a closed and bounded subset of $\mathbb{R}^{p}$, then the intervals can be chosen uniformly. Examples of compact manifolds are the circle $S^{1}$, sphere $S^{2}$, generalised tori and the Lie groups of orthogonal matrices $S O(n)$; however $\mathbb{R}^{n}$ and $S E(n), n \geq 1$, are not compact.

Completely classifying differentiable functions under $\mathcal{A}$-equivalence is intractable, but the problem may be simplified by aiming to classify only those functions (or germs) whose equivalence class is in some sense large. The simplest case is to find classes of functions (or their singularities) that are $\mathcal{A}$-stable, ie such that there exists a neighbourhood in $C^{\infty}(M, N)$ of $\mathcal{A}$-equivalent functions 
(or exhibiting the same singularity type). In other words, there is no qualitative change under sufficiently small perturbations.

This is illustrated by the following theorem characterising stability for real-valued functions. The derivative of a differentiable function $f: M \rightarrow \mathbb{R}$ on an $n$-dimensional manifold $M$ is represented by the gradient vector of a local representative which is a $1 \times n$ matrix; it has either rank 1 at a regular point or rank 0 at a singularity. Therefore, the Taylor series expansion of the representative at a singularity must begin with second-order terms.

Theorem 4.3. Let $M$ be a compact manifold of dimension $n$. Then a differentiable function $f: M \rightarrow \mathbb{R}$ is $\mathcal{A}$-stable if and only if:

1. it has at most finitely many singularities and

2. if $x \in M$ is a singularity then there is local representative of $f$ of the form

$$
\left(x_{1}, \ldots, x_{n}\right) \mapsto-\sum_{i=1}^{j} x_{i}^{2}+\sum_{i=j+1}^{n} x_{i}^{2}
$$

for some index $0 \leq j \leq n$ and

3. if $x \neq y \in M$ are singularities, then $f(x) \neq f(y) \in \mathbb{R}$.

The $n+1$ possible functions defined by (26) are local normal forms with respect to $\mathcal{A}$-equivalence for singularities $\mathbb{R}^{n} \rightarrow \mathbb{R}$. If the Hessian of $f$ at a singularity $x$ (symmetric matrix of second-order partial derivatives) is non-singular, then it has a normal form like this. The forms are 2 -jets and in fact they are 2-determined. In the case $n=2$, they correspond to models for stable local minima, saddle points and local maxima, for $j=0,1,2$ respectively. This is a local property of the function, whereas the last condition is a global property.

In addition to stability, singularity theory frequently seeks properties $\mathcal{P}$ that are exhibited by almost all functions, for example, that a function only possess singularities coming from a prescribed list. 'Almost all', in $C^{\infty}(M, N)$, means 'in an open and dense subset' or, when $M$ is non-compact, 'in a countable intersection of open, dense subsets', which is called a residual set. It is a theorem that in the Whitney $C^{\infty}$ topology, residual sets are in fact dense so that at least part of the strength of the compact case is retained. Openness ensures that sufficiently small perturbations of a $\mathcal{P}$-function retain $\mathcal{P}$, while density means there exists an arbitrarily small perturbation of a non- $\mathcal{P}$-function that has $\mathcal{P}$. Such a property $\mathcal{P}$ is called generic in either case. It is important to emphasise that the term 'generic' is not a description of functions themselves but of properties of functions. Nevertheless, in practice, a specific property is frequently identified and the term is applied to the functions satisfying that property.

The key tools for establishing genericity are transversality theorems. Recall from Section 2.4 that transversality at a given point is established by a spanning condition or equivalently the nonvanishing of a determinant. If the function is perturbed a sufficiently small amount, one would expect the determinant to remain non-zero. A non-zero function on a non-compact manifold may, of course, take arbitrarily small values, however the fine control provided by the Whitney topology ensures the following fundamental theorem:

Theorem 4.4 (Thom Transversality Theorem). Let $M, N$ be differentiable manifolds and $Q$ a closed submanifold of $J^{k}(M, N)$ for some $k \geq 0$. The set $T_{Q}=\left\{f \in C^{\infty}(M, N): j^{k} f\right.$ $\left.\Phi\right\}$ is open and dense in the Whitney $C^{\infty}$ topology. 
A multijet version of this theorem also holds true, where $Q$ is taken to be a closed submanifold of $J_{r}^{k}(M, N)$. Part 3 of Theorem 4.3 can be proved by taking $r=2$ and considering the submanifold $S$ corresponding to pairs of singular 1-jets. Since it has codimension $2 n+1$, transversality is only possible if $j_{2}^{1} f(\mathbf{x}, \mathbf{y}) \notin S$ for any distinct pair $\mathbf{x}, \mathbf{y} \in \mathbb{R}^{n}$.

If $M$ is not compact and $Q$ not closed then the statement of Theorem 4.4 must be weakened to say that $T_{Q}$ is a residual set. For a serial robot manipulator, the jointspace is compact so long as the joints are revolute but may not be otherwise. However there are many standard cases in which the relevant submanifold $Q$ may not be closed. In some cases, one is interested in simultaneous transversality to a collection of submanifolds, for example the corank $r$ submanifolds in Section 4.2. A collection $\mathcal{S}$ of submanifolds of a manifold $M$ is called a stratification (and the submanifolds strata) if some neighbourhood of any point of $M$ intersects at most finitely many strata and if a stratum $Y$ intersects the closure of another $X$, then $Y \subset X$. If, additionally, a certain technical condition, Whitney regularity, concerning the tangent spaces to strata such as $X$ and $Y$, then the full strength of the Thom Transversality Theorem can be recovered in respect of functions simultaneously transverse to all the strata: the set $T_{\mathcal{S}}$ of transverse functions is both open and dense. ${ }^{78}$

While the Thom Transversality Theorem is a powerful tool in singularity theory, it has some limitations in applications to robot kinematics. The theorem concerns the space of all differentiable functions between the relevant manifolds. That is far too general for robot kinematic mappings. Indeed, for serial manipulators, the kinematic mappings represented by the product of exponentials formalism (17) form only a finite-dimensional family of functions-Denavit-Hartenberg parameters can be used as an explicit parametrisation. The following theorem generalises the Elementary Transversality Theorem and provides a tool for handling the case of a finite-dimensional parameter space.

Theorem 4.5. Let $M, N$ and $B$ be differentiable manifolds and $Q$ a submanifold of $J^{k}(M, N)$ for some $k$. Let $F: M \times B \rightarrow N$ and $F_{b}: M \rightarrow N$ be as in Theorem 2.6 and define $\Phi: M \times B \rightarrow$ $J^{k}(M, N)$ by $\Phi(x, b)=j^{k} F_{b}(x)$. If $\Phi \pitchfork Q$ then $\left\{b \in B: j^{k} F_{b} \pitchfork Q\right\}$ is dense. If $Q$ is also closed then this set is open in $B$.

In specific cases, checking the transversality condition on $\Phi$ may be tricky. However, if $B$ has large enough dimension it may be possible to ensure that $\Phi$ is a submersion - that its derivative is surjective (and hence non-singular) - everywhere. In that case it is trivially transverse to any submanifold of the jet bundle.

Returning once more to the local classification of singularities, note that the fibre $J^{k}\left(\mathbb{R}^{m}, \mathbb{R}^{n}\right)_{(0,0)}$ of the bundle approximates the set of germs in the same way that the jet bundle approximates the space of all smooth functions. $\mathcal{A}$-equivalence (global or local) can be restricted to equivalence of $k$-jets in an obvious way: the diagram (21) should commute for the corresponding $k$-jets. The coordinate changes are now effectively jets, so belong to a finite-dimensional manifold which is, in fact, a Lie group. An equivalence class in $J^{k}(M, N)$ (respectively $\left.J^{k}\left(\mathbb{R}^{m}, \mathbb{R}^{n}\right)_{(0,0)}\right)$ may not correspond to a single class in $C^{\infty}(M, N)$ (respectively $\left.C^{\infty}\left(\mathbb{R}^{m}, \mathbb{R}^{n}\right)_{(0,0)}\right)$. However if it does, then any function (or germ) whose $k$-jet lies in the class is called $k$-determined or, if such a $k$ exists, finitely determined. This leads to an algorithmic approach for generating such classifications, by means of the method of complete transversals. ${ }^{81}$

This is connected to one further valuable paradigm from singularity theory. For any family of smooth mappings a key role is provided by the bifurcation set of parameters for which the corresponding mapping exhibits a non-stable singularity. For example, for a $p$-parameter family of 
kinematic mappings $F: \Theta \times \mathbb{R}^{p} \rightarrow S E(3)$ the bifurcation set comprises the set of design parameters $w \in \mathbb{R}^{p}$ for which the corresponding kinematic mapping exhibits a non-stable singularity type, representing the boundary between qualitatively different types of kinematic behaviour. One thinks of the family as a deformation of the kinematic mapping for a particular choice of parameter values. Then, so long as the singularity classification satisfies some regularity properties, such as Whitney regularity, the theory determines the local structure of the bifurcation set up to diffeomorphism. More precisely, provided its $k$-jet is transverse to the classification in the $k$-jet bundle, then the bifurcation set is diffeomorphic to the bifurcation set of a minimal versal deformation at a normal form. Here, versal is an algebraic interpretation of jet transversality using the germ structure, while minimality means that the sum of dimensions on the left-hand side in the transversality condition (10) are equal to that of the right-hand side. Finding nice forms for minimal versal unfoldings is a natural extension of the concept of normal forms. The same idea is used by Donelan and Gibson ${ }^{76}$ to provide versal deformations for screw systems. As a result, it is possible to determine adjacency or specialisation diagrams for the classification.

\section{Applications to Robot Manipulators}

In this section, the ideas of the previous sections are explicitly brought together in the service of exploring robot kinematic singularities. Four key sets of results are described concerning firstorder properties of kinematic mappings in Sections 5.1 and 5.2, general classification of kinematic mapping singularities in Section 5.3 and finally singularities of trajectories in Section 5.4.

\subsection{Singularities of Fixed Rank}

As noted above in Section 4.2, singularities of fixed corank can be encoded as a submanifold of the 1-jet bundle. Usually, in robot kinematics, one is interested in the case $N=S E(3)$ and so $n=6$, or possibly $N=S O(3)$ or $\mathbb{R}^{3}$ with $n=3$ if only rotational or translational capacity is being considered. Suppose that $f: \Theta \rightarrow S E(3)$ (or $f: \Theta \rightarrow G$ where $G$ is some subgroup of $S E(3)$, such as $\left.S O(3), \mathbb{R}^{3}\right)$ is a kinematic mapping and $\operatorname{dim} \Theta=m$. Pai and Leu ${ }^{35}$ call $f$ generic or, more precisely, one-generic (see Section 4.3 concerning use of the term 'generic') if $j^{1} f \pi^{r}$ for all $r \geq 0$. If $G=S E(3)$ and $j^{1} f \mp \Sigma^{r}$, that is $f$ is 1 -generic, then the inequality (25) determines that $m \geq 4$ is necessary for $\Sigma^{1} f \neq \emptyset, 6 \leq m \leq 8$ is necessary for $\Sigma^{2} f \neq \emptyset$ and for all $r \geq 3$ and for all $m$, $\Sigma^{r} f=\emptyset$. Similar inequalities can be derived when $n=3$.

The codimension of the singularity set has practical significance. If the codimension is 1 then the singularity set, at least locally, separates configurations so that there exist a pair of non-singular configurations that cannot be joined by a continuous path (a non-removable singularity in Karger's terminology ${ }^{42}$ ). On the other hand, if the codimension $\geq 2$ then it is avoidable (or removable) in the sense that for any two non-singular configurations in a connected component of $\Theta$, there will be a continuous path joining them. The only case for spatial kinematics in which the singular set $\Sigma^{1}$ has codimension 1 is when $m=6$.

The Thom Transversality Theorem asserts that, for a given jointspace $\Theta$, almost all functions $\Theta \rightarrow S E(3)$ are one-generic; but it does not follow that this is true for the finite-dimensional family of kinematic mappings of serial manipulators, for the reason discussed towards the end of Section 4.3. Instead, one must attempt to apply Theorem 4.5 and hence must be able to establish transversality for the family denoted $\Phi$. Pai and Leu ${ }^{35}$ focus on the class of wrist-partitioned 
manipulators (see Section 1) and consider separately orientation and translation singularities of the 3-dof sub-mechanisms. In these dimensions only corank 1 singularities can occur transversely. Pai and Leu determine geometric conditions under which transversality would hold for some specific architectures but they do not address directly whether the conditions hold for a large class of such manipulators, i.e. truly generically. That question is resolved in the most general case, where the individual joints are allowed to be revolute, prismatic or helical, by the following theorem.

Theorem 5.1. For any $m \geq 1$, there is an open and dense set of general $m$-joint serial manipulators (architecture class $\mathcal{O}$ ) whose kinematic mapping has 1-jet extension transverse to the submanifolds $\Sigma^{r} \subset J^{1}\left(\mathbb{R}^{m}, S E(3)\right)$ for all $0 \leq r \leq \min \{m, 6\}$.

Proof. Let $B=(\mathfrak{s e}(3)-\{\mathbf{0}\})^{m}$ be the set of $m$-tuples of non-zero twists. Consider the parametrised family of functions $F: \mathbb{R}^{m} \times B \rightarrow S E(3)$, defined by

$$
F\left(\boldsymbol{\theta}, \mathbf{u}_{1}, \ldots, \mathbf{u}_{m}\right)=e^{\theta_{1} \mathbf{u}_{1}} e^{\theta_{2} \mathbf{u}_{2}} \cdots e^{\theta_{m} \mathbf{u}_{m}} .
$$

Here $\boldsymbol{\theta}=\left(\theta_{1}, \ldots, \theta_{m}\right) \in \mathbb{R}^{m}$ and $\mathbf{u}_{i}=\left(u_{i 1}, \ldots, u_{i 6}\right) \in \mathfrak{s e}(3)-\{\mathbf{0}\}, 1 \leq i \leq m$, with respect to some basis for $\mathfrak{s e}(3)$. The family contains representatives of all serial manipulator kinematic mappings. The function $\Phi: \mathbb{R}^{m} \times B \rightarrow J^{1}\left(\mathbb{R}^{m}, S E(3)\right)$, as defined in Theorem 4.5, can be written as:

$$
\left(\boldsymbol{\theta}, \mathbf{u}_{1}, \ldots, \mathbf{u}_{m}\right) \mapsto\left(\boldsymbol{\theta}, F\left(\boldsymbol{\theta}, \mathbf{u}_{1}, \ldots, \mathbf{u}_{m}\right), D F_{\left(\mathbf{u}_{1}, \ldots, \mathbf{u}_{m}\right)}(\boldsymbol{\theta})\right) .
$$

Since the tangent space to each of the submanifolds $\Sigma^{r}$ projects onto the base $\mathbb{R}^{m} \times S E(3)$ of the jet bundle, it is only necessary to ensure the transversality condition holds on projection to the fibres. Now the derivatives of $F$ with respect to $u_{i j}, 1 \leq i \leq m, i \leq j \leq 6$ span the fibre $L\left(\mathbb{R}^{m}, \mathfrak{s e}(3)\right)$ and hence transversality is automatic.

One would like to be able to make similar assertions in the cases of architecture classes $\mathcal{R}$ and $\mathcal{R} \mathcal{P}$. However, in these cases, the twists $\mathbf{u}_{i}$ in the formula (27) must be restricted to the relevant subsets of the Lie algebra $\mathfrak{s e}(3)$, namely the quadric cones $Q_{0}$ and $Q_{\infty}$ defined by the vanishing of the fundamental Klein and Killing invariants respectively. While $Q_{0}$, the classical Klein quadric of line geometry, is a 5 -dimensional hypersurface, the Killing quadric $Q_{\infty}$ is degenerate and is simply the 3-dimensional subspace given by $\boldsymbol{\omega}=0$. Differentiation with respect to design parameters for the given class, as in the proof of Theorem 5.1, can only be performed along the tangent spaces to the relevant quadric at each twist $\mathbf{u}_{i}$. For $Q_{0}$, this is precisely the subspace spanned by the reciprocal screw system to the screw spanned by $\mathbf{u}_{i}$, while since $Q_{\infty}$ is linear it is its own tangent space. Transversality is therefore a more subtle issue and the question of genericity remains open for these classes.

In what follows some specific theory is developed towards answering these questions and is applied to the example of 6-dof wrist-partitioned manipulators. The formula (18), derived above from the Campbell-Baker-Hausdorff formula, provides a succinct way of expressing the jet of a serial manipulator kinematic mapping, and hence enabling more precise statements about transversality to be made. Expanding $f\left(\theta_{1}+\alpha_{1}, \ldots, \theta_{m}+\alpha_{m}\right)$ about $\boldsymbol{\alpha}=\mathbf{0}$, differentiating, and using 
exponential coordinates gives:

$$
\begin{array}{r}
j^{1} f(\boldsymbol{\theta})=\left(\boldsymbol{\theta}, f(\boldsymbol{\theta}), X_{1}+\frac{1}{2} \sum_{j>1}\left[X_{1}, X_{j}\right] \theta_{j}+O(2), \ldots,\right. \\
X_{i}-\frac{1}{2} \sum_{j<i}\left[X_{i}, X_{j}\right] \theta_{j}+\frac{1}{2} \sum_{j>i}\left[X_{i}, X_{j}\right] \theta_{j}+O(2), \\
\left.\ldots, X_{m}-\frac{1}{2} \sum_{j<m}\left[X_{m}, X_{j}\right] \theta_{j}+O(2)\right)
\end{array}
$$

As in equation (18), $O(2)$ here indicates terms that are of quadratic or higher order in the variables $\theta_{i}$ - they also involve higher-order brackets of the $X_{i}$. In view of (28), it is clear that $j^{1} f(\mathbf{0}) \in \Sigma^{r}$ precisely when the corresponding set of screws $\left(X_{1}, \ldots, X_{m}\right)$ span a $(m-r)$-system for $m \leq 6$ or a $(6-r)$-system for $m>6$.

Lerbet and $\mathrm{Hao}^{36}$ implicitly obtain the form (28) in their Appendix and use it to establish a necessary condition for transversality, equivalent to one part of Lemma 4.2. Suppose $f$ is the kinematic mapping of a $m$-dof serial manipulator, written in product-of-exponentials form. If $\boldsymbol{\theta}=\mathbf{0} \in \Sigma^{r} f$ then the twists $X_{1}, \ldots, X_{m}$ span a $q$-system where $q-\operatorname{rank} T_{\mathbf{0}} f=\min \{m, 6\}-r$. The derivatives of $j^{1} f$ with respect to $\theta_{\ell}, 1 \leq \ell \leq m$, evaluated at $\boldsymbol{\theta}=\mathbf{0}$ (and restricted to the fibre over the source $\mathbf{0}$ and target $f(\mathbf{0})$ ) can be written as $6 \times m$ matrices:

$$
\Upsilon_{\ell}=\frac{1}{2}\left(\begin{array}{lllllll}
{\left[X_{1}, X_{\ell}\right]} & \cdots & {\left[X_{\ell-1}, X_{\ell}\right]} & 0 & -\left[X_{\ell+1}, X_{\ell}\right] & \cdots & \left.-\left[X_{m}, X_{\ell}\right]\right)
\end{array}\right.
$$

Hence the question of transversality is locally determined by whether, after 'restriction and projection', these $m$ matrices span the $(m-q)(6-q)$-dimensional subspace identified in Lemma 4.2. It is clear that in order to meet this condition, the columns of the $m$ derivative matrices $\Upsilon_{\ell}$ must span a complement to the screw system generated by $X_{1}, \ldots, X_{m}$ in $\mathfrak{s e}(3)$; in other words, the subalgebra generated by these twists must be the whole algebra. Lerbet and Hao observe that in the case $m=6$ and $q=5$ this condition is automatically satisfied since there are no 5-dimensional Lie subalgebras of $\mathfrak{s e}(3)$. However that is not sufficient to ensure transversality.

Example: wrist-partitioned 6-dof serial manipulators. These were introduced briefly in Section 1. Lipkin and Pohl ${ }^{79}$ enumerate the types of singularity that these and other types of serial manipulator can undergo and provide a simple representation of the Jacobian. Tchon and Muszynski ${ }^{33}$ provide an analysis of PUMA-type arms of this sort; Hayes et all $l^{15}$ provide an analysis of the singularities that arise for another example, the KUKA KR-15/2. The general architecture is that there is a 3 -dof regional manipulator, whose joints are revolute and usually perpendicular or parallel as this simplifies the forward kinematics considerably. The wrist centre $W$ is some point in the end-effector of the regional manipulator and the wrist-component is a 3 -dof spherical mechanism centred at $W$, so that its three revolute axes intersect at this point. From the Lie algebra point of view, the kinematics can be represented by a product-of-exponentials formula (17) in which twists $X_{4}, X_{5}, X_{6}$ belong to a subalgebra $\mathfrak{r}$ of instantaneous rotations about a point (the wrist centre) which, if one chooses coordinates so that $W$ is the origin, is simply $\mathfrak{s o}(3)$. It is further assumed (a) that no two consecutive twists $X_{i}, X_{i+1}$ are linearly dependent, since such a manipulator would be equivalent to one of $\leq 5$-dof in which such pairs were contracted to a single joint, and (b) that $X_{3} \notin \mathfrak{r}$ since then the last 4 joints could only effect rotation about $W$. 
Hence, using the form for twists in Section 3.1, the Jacobian (1), in any given configuration, can be written as

$$
\left(\begin{array}{cccccc}
\boldsymbol{\omega}_{1} & \boldsymbol{\omega}_{2} & \boldsymbol{\omega}_{3} & \boldsymbol{\omega}_{4} & \boldsymbol{\omega}_{5} & \boldsymbol{\omega}_{6} \\
\mathbf{v}_{1} & \mathbf{v}_{2} & \mathbf{v}_{3} & \mathbf{0} & \mathbf{0} & \mathbf{0}
\end{array}\right)
$$

where for $i=1,2,3, \boldsymbol{\omega}_{i} \cdot \mathbf{v}_{i}=0$ since the joints are revolute and $\mathbf{v}_{3} \neq \mathbf{0}$ by assumption (b). If one takes a given singularity as the home configuration then it follows that $X_{1}, \ldots, X_{6}$ are linearly dependent in the Lie algebra.

As noted before, wrist-partitioned architectures therefore display two modes of singularity, (I) singularities of the wrist itself and (II) singularities relating to the positioning of the wrist-centre. Mode (I) occurs when $\operatorname{det}\left(\boldsymbol{\omega}_{4} \boldsymbol{\omega}_{5} \boldsymbol{\omega}_{6}\right)=0$ and so $X_{4}, X_{5}, X_{6}$ span a 2 -system in $\mathfrak{s e}(3)$. A 1 -system is not possible because of the assumed linear independence of consecutive twists. For mode (II) singularities, $\operatorname{det}\left(\begin{array}{lll}\mathbf{v}_{1} & \mathbf{v}_{2} & \mathbf{v}_{3}\end{array}\right)=0$ and some linear combination of $X_{1}, X_{2}, X_{3}$ lies in $\mathfrak{r}$. A mode (I) singularity is usually termed a wrist singularity. For many manipulator architectures used in practice, the equation for mode (II) factorises, giving two submodes termed shoulder and elbow singularities, corresponding to $X_{1} \in \mathfrak{r}$ and $a X_{2}+b X_{3} \in \mathfrak{r}$ for some $a, b \in \mathbb{R}$, respectively. One way to envisage mode (II) is that the 3 -system spanned by $X_{1}, X_{2}, X_{3}$ and $\mathfrak{r}$ have non-trivial intersection. In particular, it may be possible for them to intersect in a line, hence give a corank 1 singularity, or a plane, corank 2. Further, modes (I) and (II) may coincide potentially giving singularities of corank 1, 2 or 3 . See Tchon and Muszynski ${ }^{33}$ for some discussion of this.

Pure wrist singularities, i.e. mode (I) but not mode (II), occur transversely for any wristpartitioned manipulator. For suppose $\mathbf{0} \in \mathbb{R}^{6}$ is a corank 1 singularity of the kinematic mapping $f$ of this type, and $\mathbf{c}=\left(c_{1}, \ldots, c_{6}\right)^{t}$ spans $\operatorname{ker} T_{\mathbf{0}} f$. Then $c_{4} X_{4}+c_{5} X_{5}+c_{6} X_{6}=\mathbf{0}$. Moreover, $c_{4}, c_{6} \neq 0$, otherwise there are consecutive dependent twists. Equivalently, $\left[X_{4}, X_{5}\right],\left[X_{5}, X_{6}\right] \neq \mathbf{0}$. It follows that $\Upsilon_{4} \cdot \mathbf{c}=\Upsilon_{6} \cdot \mathbf{c}=\mathbf{0}$, however

$$
\Upsilon_{5} . \mathbf{c}=\frac{1}{2}\left[c_{4} X_{4}-c_{6} X_{6}, X_{5}\right]=c_{4}\left[X_{4}, X_{5}\right] \neq \mathbf{0}
$$

Now $X_{4}, X_{5}, X_{6}$ span a 2-dimensional subspace of $\mathfrak{s o}(3)$, which is a subalgebra of $\mathfrak{s e}(3)$. So $\Upsilon_{5} . \mathbf{c} \in$ $\mathfrak{s o}(3)$, but $\mathfrak{s o}(3)$ has no 2 -dimensional subalgebras, so it must span a complement to $\operatorname{im} T_{\mathbf{0}} f$ in $\mathfrak{s e}(3)$, hence $j^{1} f \pi \Sigma^{1}$ at $\mathbf{0}$.

For a pure mode (II) corank 1 singularity, given $\sum_{i=1}^{6} c_{i} X_{i}=\mathbf{0}$ then $c_{1} \mathbf{v}_{1}+c_{2} \mathbf{v}_{2}+c_{3} \mathbf{v}_{3}=\mathbf{0}$. In a similar way as for mode (I), one requires for transversality that the translational component of $c_{1}\left[X_{1}, X_{2}\right]$, namely $c_{1}\left(\boldsymbol{\omega}_{1} \times \mathbf{v}_{2}-\boldsymbol{\omega}_{2} \times \mathbf{v}_{1}\right)$, span the complement to the span of $\mathbf{v}_{1}, \mathbf{v}_{2}, \mathbf{v}_{3}$ in $\mathbb{R}^{3}$. In particular, a necessary condition is $c_{1} \neq 0$. That is not ruled out a priori (for example $c_{1}=0$ at a shoulder singularity) so transversality may fail.

However, now incorporate design parameters (eg Denavit-Hartenberg parameters) into the problem. Suppose these parameters $\mathbf{u}_{i}=\left(u_{i 1}, \cdots, u_{i r}\right)$ for the $(i-1)$ st link and $i$ th with $M_{i}(\mathbf{0})=I$. Then, following Brockett, ${ }^{71}$ the kinematic mapping has the form, cf (17):

$$
F\left(\boldsymbol{\theta}, \mathbf{u}_{i}\right)=M_{i} \cdot e^{\theta_{1} M_{1}^{-1} X_{i} M_{i}} \cdots e^{\theta_{i-1} M_{i}^{-1} X_{i-1} M_{i}} \cdot e^{\theta_{i} X_{i}} \cdots e^{\theta_{6} X_{6}}
$$

This is amenable to application of Theorem 4.5. The terms $X_{\ell}, \ell<i$, in the 1-jet extension (28) are replaced by $M_{i}^{-1} X_{i-1} M_{i}$. Differentiation with respect to the design parameters yields, by (14), matrices of the form

$$
\left(\begin{array}{llllll}
{\left[Y_{i}^{j}, X_{1}\right]} & \cdots & {\left[Y_{i}^{j}, X_{i-1}\right]} & \mathbf{0} & \cdots & \mathbf{0}
\end{array}\right)
$$


where $Y_{i}^{j}=\partial M_{i} / \partial u_{i j}(\mathbf{0})$, for $1 \leq j \leq r$. Details of this approach, and the circumstances under which the additiional matrices enable the hypotheses of Theorem 4.5 to be satisfied, must be the subject of subsequent work.

\subsection{Screw System Genericity}

While one-genericity addresses manipulator singularities at a fundamental level, it does not fully capture the capability or loss of capability of the motion, in particular rotational and translational capabilities. In a given configuration, these are determined by the screw system. For a manipulator with $m$ joints, typically the instantaneous motion is described by an $m$-system, but this will contract to an $r$-system, $r<m$ at a singularity. Gibson and Donelan ${ }^{76}$ prove that the hierarchy of screw systems described in Section 3.3 forms a Whitney regular stratification of the Grassmannian manifold of $k$-dimensional subspaces of $\mathfrak{s e}(3)$. For $m \leq 3$, where singularities can be avoided, it follows from the stratified version of the Transversality Theorem that for an open dense set of kinematic mappings the 1-jet is transverse to the screw system stratification. However for $m>3$, where corank 1 or 2 singularities may occur generically, the $r$-screw system stratifications would need to be sewn together and the resulting stratification of the jet bundle shown to be Whitney regular. This seems likely to be the case even as the classification stands; if not then there is a procedure for refining a given stratification to a regular one. ${ }^{80}$

For serial manipulators, the argument in the proof of Theorem 5.1 ensures that architecture class $\mathcal{O}$ has the property of screw system genericity for $m \leq 3$. However, once again, restricting to revolute joints only results in failure of genericity. Cocke et $a l^{82}$ prove the following:

Theorem 5.2. If a 3-dof kinematic mapping $f$ possesses a type IIA system, with pitch modulus $h=0$, then that remains true for kinematic mappings in some (Whitney) open neighbourhood of $f$.

Since the codimensions of these classes is greater than 3, they cannot be intersected transversely so screw system genericity fails. In particular this holds true for $3 \mathrm{R}$ serial manipulators. Note however that the corresponding geometry is special: type IIA occurs when three non-coplanar revolute joints intersect in a point and hence corresponds to a rotational manipulator (spherical wrist). A similar theorem holds also for type IIB 3-systems with principal pitch zero, a class of codimension 6. Geometrically, the three revolute joints must be non-copunctual but coplanar. However in that case the transversality condition fails, meaning that the singular phenomenon is sensitive to the design parameters of the mechanism.

\subsection{Normal Forms for Serial Manipulator Kinematics}

Tchon $^{26,27}$ determines a list of local normal forms for singularities for serial manipulators using classical results and methods of singularity theory. ${ }^{68}$ He states results for $m$-dof manipulators for $m \geq 2$, but here just the particular case $m=6$ is given.

Theorem 5.3. If a 6-dof serial manipulator has forward kinematic mapping $f: \Theta \rightarrow S E(3)$ that belongs to a certain residual subset of $C^{\infty}(\Theta, S E(3))$, then in the neighbourhood of any point $\theta \in \Theta$, there are parametrisations $\left(x_{1}, \ldots, x_{6}\right)$ of a neighbourhood of $\theta$ and $\left(y_{1}, \ldots, y_{6}\right)$ of a neighbourhood 
of $f(\theta) \in S E(3)$, and a local representative of $f$ of the form

$$
\left(y_{1}, \ldots, y_{6}\right)=\left(x_{1}^{k}+\sum_{r=1}^{k-2} x_{r+1} x_{1}^{r}, x_{2}, \ldots, x_{6}\right)
$$

for some $k, 1 \leq k \leq 7$, or

$$
\begin{aligned}
& \left(y_{1}, \ldots, y_{6}\right)=\left(x_{1}^{2} \pm x_{2}^{2}+x_{3} x_{1}+x_{4} x_{2}, x_{1} x_{2}, x_{3}, \ldots, x_{6}\right) \\
& \left(y_{1}, \ldots, y_{6}\right)=\left(x_{1}^{2} \pm x_{2}^{2}+x_{3} x_{1}+x_{4} x_{2}+x_{5} x_{2}^{2}, x_{1} x_{2}, x_{3}, \ldots, x_{6}\right) \\
& \left(y_{1}, \ldots, y_{6}\right)=\left(x_{1}^{3}+x_{2}^{2}+x_{3} x_{1}+x_{4} x_{2}+x_{5} x_{1}^{2}+x_{6} x_{2}^{2}, x_{1} x_{2}, x_{3}, \ldots, x_{6}\right) \\
& \left(y_{1}, \ldots, y_{6}\right)=\left(x_{1}^{3}+x_{3} x_{1}+x_{4} x_{2}+x_{5} x_{1} x_{2}, x_{1}^{2}+x_{2}^{2}+x_{6} x_{1}, x_{3}, \ldots, x_{6}\right)
\end{aligned}
$$

Note that the form (34), $k=1$ is the non-singular case, $2 \leq k \leq 7$ have corank 1 singularities and the cases in (35) are corank 2.

However, as Tchoń notes, while the normal forms can be regarded as generic for functions in general, the kinematic mappings of serial manipulators have special form, so there is no guarantee that the forms will apply to any actual kinematic singularities at all! In subsequent papers, ${ }^{30,31,33,34}$ he determines actual normal forms for a number of standard robot manipulators such as the PUMA. In so doing he proves that the PUMA possesses corank 1 Morse shoulder and elbow singularities, that is type (34) with $k=2$. However wrist singularities are highly degenerate in this sense (infinite codimension) and the kinematic mapping lies outside the residual set of Theorem 5.3.

\subsection{Trajectory Singularities}

The kinematic mapping for a robot manipulator determines the motion of its end effector or platform in terms of its joint variables. Associated to this motion is the family of trajectories of each of the points in the end-effector/platform. One may be interested in isolating one point whose trajectory has some specific characteristic. This was frequently the aim in the classical design of mechanism, where features such as dwell, return and straight-line approximation were desirable features, corresponding to specific singularities or curvature properties of trajectories (see for example $\mathrm{Hunt}^{3}$ ). In robotics, examples are the choice of centre in the a remote centre compliance device $^{83,84}$ and the determination of wrist centre in a wrist-partitioned serial manipulator. ${ }^{14,19}$ So, from the design perspective, one is interested in the whole family of trajectories.

To place this in the singularity theory context described here, consider the kinematic mapping $f: \Theta \rightarrow S E(3)$ of the end-effector of a manipulator. Given a point $\mathbf{w} \in \mathbb{R}^{3}$, in some system of coordinates in the end-effector, the trajectory of $\mathbf{w}$ is given by composing the action of the Euclidean isometry group on $\mathbf{w} \in \mathbb{R}^{3}$ (sometimes called the evaluation map on $\mathbf{w}$ ) with the kinematic mapping:

$$
\tau_{w}: \Theta \rightarrow \mathbb{R}^{3}, \quad \tau_{w}(x)=f(\boldsymbol{\theta}) . \mathbf{w} .
$$

The trajectories form a family, parametrised by $\mathbf{w} \in \mathbb{R}^{3}$ :

$$
\tau: M \times \mathbb{R}^{3} \rightarrow \mathbb{R}^{3} ; \quad \tau(x, \mathbf{w})=\tau_{w}(x) .
$$

In any given configuration $\boldsymbol{\theta} \in \Theta$ there may be points $\mathbf{w}$ whose trajectories are at a singularity, whether or not the kinematic mapping itself has a singularity at $\boldsymbol{\theta}$ (in fact, if $f$ is singular then all 
trajectories must also be singular). The simplest intuition here is that for 1-dof planar motions there is typically an instantaneous centre of rotation. That would be the only singular trajectory at that instant but there would be a locus of instantaneous centres, namely the centrode. In 1-dof spatial motions, there is typically an instantaneous axis of rotation, but unless its pitch is zero, points on the axis are not stationary so there are no singular trajectories, whereas if the pitch is zero then all points on the axis have a singular trajectory at that instant. This generalises to multi-dof motions as follows. Given a kinematic mapping $f: \Theta \rightarrow S E(3)$, its instantaneous singular set (ISS) of $f$ at $\boldsymbol{\theta} \in \Theta$ is

$$
I_{f, \boldsymbol{\theta}}=\left\{\mathbf{w} \in \mathbb{R}^{3}: \tau_{f, w} \text { is singular at } \boldsymbol{\theta}\right\} .
$$

If $\operatorname{dim} \Theta=k$ then $f$ is a $k$-dimensional family of ISSs.

For example, for a $3 \mathrm{R}$ regional manipulator, the ISS in a given configuration is precisely the union of singular surfaces identified by Stanišić and Engelberth. ${ }^{14}$ Indeed, they characterize these surfaces in terms of the principal pitches of the associated 3-system and their classification can be derived from the following more general theorem: ${ }^{85}$

Theorem 5.4. Let $f: \Theta \rightarrow S E(3)$ be a kinematic mapping with rank $k=\operatorname{dim} \Theta$ at $\boldsymbol{\theta} \in \Theta$ and let $S$ be the associated screw system. For a point $\mathbf{w} \in \mathbb{R}^{3}$ let $A_{w}$ be the $\alpha$-plane in the Klein quadric $Q_{0}$ representing the bundle of lines through $\mathbf{w}$. Then $\mathbf{w} \in I_{f, \boldsymbol{\theta}}$ if and only if $S$ intersects $A_{w}$ in a space of dimension $\geq \max (1, k-2)$. The dimension of the intersection is precisely the corank of the singularity of the trajectory of $\mathbf{w}$.

This follows by applying the Chain Rule to $\tau_{f, w}$, viewed as a composition of the evaluation map with the kinematic mapping. An important corollary is that the ISS is the union of all the lines corresponding to points of $S \cap A_{w}$ satisfying the condition of the theorem. It is a straightforward exercise to describe the ISS associated to a given screw system. For example, in the simplest case of a IA 13 -system (see Table 1) the ISS is an elliptic single-sheeted hyperboloid (or empty if all principal pitches have the same sign) and each point on it has a singularity of corank 1.

For type IIA, $h=0$, corresponding to an $\alpha$-plane of lines through a given point in $\mathbb{R}^{3}$, every point is singular but the given point has a corank 3 singularity. From the point of view of singularity theory, this is highly non-generic: corank 3 singularities of functions from $\mathbb{R}^{3} \rightarrow \mathbb{R}^{3}$ have codimension 9 in the jet bundle so could not be encountered transversely, even in a 3 -parameter family of such trajectories. However, as cited in Theorem 5.2, among kinematic mappings of class $\mathcal{R}$ where the three joints are constrained to be revolute, such screw systems, and hence highly singular trajectories are in fact stable.

Gibson et $a l^{50-57}$ establish an extensive set of results concerning the classification of trajectory singularities for planar and spatial motions with up to 3 dof. They make extensive use of the method of complete transversals. This relates to the above theory of ISSs in the same way that Tchon's classification relates to the analysis of rank of a singularity. The key theorem, due to Gibson and $\operatorname{Hobbs}^{50}$ concerns the family of kinematic mappings $C^{\infty}(\Theta, S E(3))$, not restricted to any particular class of manipulators. For any $r$, there is an induced jet extension

$$
j_{1}^{r} \tau: \Theta \times \mathbb{R}^{3} \longrightarrow J^{r}\left(\Theta, \mathbb{R}^{3}\right)
$$

where the subscript 1 indicates we are taking jets with respect to the first component only, which represents the $r$-jets of all the trajectories at the same time. The following theorem asserts that for almost all kinematic mappings, every trajectory will only exhibit typical singularities. 
Theorem 5.5. Let $\mathcal{S}$ be a finite or Whitney regular stratification of $J^{r}\left(\Theta, \mathbb{R}^{3}\right)$. The set of kinematic mappings $f: \Theta \rightarrow S E(3)$ with $j_{1}^{r} \tau$ transverse to $\mathcal{S}$ is residual in $C^{\infty}(\Theta, S E(3))$, endowed with the Whitney $C^{\infty}$ topology. A multijet version also holds.

In practice, the stratification is likely to arise from an $\mathcal{A}$-classification of singularities. The transversality condition means that only singularity types up to codimension $k=\operatorname{dim} \Theta$ occur generically. Moreover, it also implies that the bifurcation sets for the non-stable singular trajectories must have a standard form. However the lists of types do become quite large for $k=3$ in the spatial case and hence tend to lose informative value. Moreover, the theorem relates to the whole class of mappings, whereas, as has been emphasised before, in practice one is interested in specific finite-dimensional classes determined by some architecture and as the example above illustrates, in these cases highly degenerate trajectory singularities may occur stably.

\section{Conclusion}

Singularity-theoretic methods have made a valuable contribution to understanding manipulator kinematics, not least by providing a language for classifying and analysing their singularities. To some extent the natural emphasis of singularity theory on the generic and stable examples is at odds with engineering practice, where frequently it is the special case that is of value. Powerful general results concerning smooth functions may not translate to the specific types of kinematic mapping associated to standard robot manipulator architectures. Nevertheless, results such as Theorem 5.1 suggest that it may be possible to specialise the results in some cases. Further research on specific classes of manipulator and extension of the techniques described in Section 5.1 will provide focus on the right questions to ask and should promote new directions of in both singularity theory and kinematics. To start with, this will include obtaining a more complete understanding of serial manipulator singularities, their relation to the Lie subalgebra associated to the generating twists, and the bifurcations the loci undergo in relation to the design parameters. Application of deformation methods will provide robust local models for these. Recent research on the cuspidal manipulators clearly suggests the link to classical singularity theory.

The domains of parallel and wire manipulators, beyond the classical case of 1-dof mechanisms, is virtually untouched from this perspective and offer considerable scope for understanding the types of singularity that can occur. Yet there is a wealth of engineering literature on these singularities and they raise fascinating questions because of the distinction between type I and type II singularities, reminiscent of applications of singularity theory to the theory of bifurcations. Robot kinematics therefore provides one of the most interesting fields for applying singularity-theoretic methods.

\section{References}

[1] D. E. Whitney, Resolved Motion Rate Control of Manipulators and Human Prostheses, IEEE Trans. Man-Machine Systems, 10 (1969) 47-53

[2] R. Featherstone, Position and Velocity Transformations Between Robot End-Effector Coordinates and Joint Angles, Int. J. Robotics Research, 2 (1983) 35-45

[3] K. H. Hunt, Kinematic Geometry of Mechanisms, Clarendon Press, Oxford, 1978 
[4] K. Sugimoto, J. Duffy and K. H. Hunt, Special Configurations of Spatial Mechanisms and Robot Arms, Mechanism and Machine Theory, 17 (1982) 119-132

[5] K. H. Hunt, Special Configurations of Robot Arms via Screw Theory-Part I: The Jacobian and its Matrix Cofactors, Robotica, 4 (1986) 171-179

[6] K. H. Hunt, Special Configurations of Robot Arms via Screw Theory-Part II: Available End-Effector Displacements, Robotica, 5 (1987) 17-22

[7] F. L. Litvin and V. Parenti-Castelli, Configurations of Robot Manipulators and Their Identification and the Execution of Prescribed Trajectories, Trans. ASME J. Mechanisms, Transmissions and Automation in Design, 107 (1985) 170-188

[8] F. L. Litvin, Z. Yi, V. Parenti-Castelli and C. Innocenti, Singularities, Configurations and Displacement Functions for Manipulators, Int. J. Robotics Research, 5 (1986) 66-74

[9] S. L. Wang and K. J. Waldron, A Study of the Singular Configurations of Serial Manipulators, Trans. ASME J. Mechanisms, Transmissions and Automation in Design, 109 (1987) 14-20

[10] C. Gosselin and J. Angeles, Singularity Analysis of Closed-Loop Kinematic Chains, IEEE Trans. Robotics and Automation, 6 (1990) 281-290

[11] J. P. Merlet, Singular Configurations of Parallel Manipulators and Grassmann Geometry, Int. J. Robotics Research, 8 (1992) 45-56

[12] R. Di Gregorio and V. Parenti-Castelli, Mobility Analysis of the 3-UPU Parallel Mechanism Assembled for a Pure Translational Motion, Proc. IEEE/ASME Int. Conf. on Advanced Intelligent Mechatronics, Atlanta, Georgia, (1999) 520-525

[13] D. Zlatanov, I. A. Bonev and C. M. Gosselin, Constraint Singularities of Parallel Mechanisms, Proc. IEEE Int. Conf. Robotics and Automation, (2002) 496-502

[14] M. M. Stanišić and J. W. Engelberth, A Geometric Description of Manipulator Singularities in Terms of Singular Surfaces, Proc. 1st Int. Workshop on Advances in Robot Kinematics, Ljubljana, Slovenia, (1988) 132-141

[15] M. J. D. Hayes, M. L. Husty, and P. J. Zsombor-Murray, Singular Configurations of WristPartitioned 6R Serial Robots: A Geometric Perspective for Users, Trans. Canadian Soc. Mech. Eng., 26 (2002) 41-55

[16] J. W. Burdick, A Classification of 3R Regional Manipulator Singularities and Geometries, Mechanism and Machine Theory, 30 (1995) 71-89

[17] P. Wenger and J. El Omri, Changing Posture for Cuspidal Robot Manipulators, Proc. IEEE Int. Conf. Robotics and Automation, (1996) 3173-3178

[18] D. Pieper and B. Roth, The Kinematics of Manipula- tors Under Computer Control, in Proc. 2nd World Congress on the Theory of Machines and Mecha-nisms, Zakopane, Poland, Vol. 2, (1969) 159-169 
[19] M. M. Stanišić and C. M. Goehler, Singular Planes of Serial Wrist-Partitioned Manipulators and Their Singularity Mertrics, Mechanism and Machine Theory, to appear (2006)

[20] C. A. Klein and C. H. Huang, Review of Pseudoinverse Control for use with Kinematically Redundant Manipulators, IEEE Trans. Systems, Man and Cybernetics, 3 (1983) 245-250

[21] T. Shamir and Y. Yomdin, Repeatability of Redundant Manipulators: Mathematical Solution of the Problem, IEEE Trans. Automatic Control, 33 (1988) 1004-1009

[22] J. Kieffer and J. Lenarčič, On the Exploitation of Mechanical Advantage Near Robot Singularities, Proc. 3rd Int. Workshop on Advances in Robot Kinematics, Ferrara, Italy, (1992) $65-72$

[23] R. P. Paul and C. N. Stevenson, Kinematics of Robot Wrists, Int. J. Robotics Research, 2 (1983) 31-38

[24] D. H. Gottlieb, Robots and Fibre Bundles, Bull. Soc. Math. Belg., 38 (1986) 219-223

[25] D. R. Baker and C. W. Wampler, On the Inverse Kinematics of Redundant Manipulators, Int. J. Robotics Research, 7 (1988) 3-21

[26] K. Tchoń, Towards a Differential Topological Classification of Robot Manipulators, in Progress in Systems and Control Theory: Robust Control of Linear Systems and Nonlinear Control, M. Kaashoek et al (eds), Birkhäuser, Boston 1990

[27] K. Tchoń, Differential Topology of the Inverse Kinematic Problem for Redundant Robot Manipulators, Int. J. Robotics Research, 10 (1991) 492-504

[28] K. Tchoń, Calibration of Manipulator's Kinematics: A Singularity Theory Approach, IEEE Trans. Robotics and Automation, 8 (1992) 671-678

[29] K. Tchoń and P. Urban, Classification of Kinematic Singularities in Planar Robot Manipulators, Systems Control Lett., 19 (1992) 293-302

[30] K. Tchoń, Normal Forms of Singular Kinematics of 3R Robot Manipulators, Applied Math. and Comp. Sci., 5 (1995) 391-407

[31] K. Tchoń, A Normal Form of Singular Kinematics of Robot Manipulators with Smallest Degeneracy, IEEE Trans. Robotics and Automation, 11 (1995) 401-404

[32] K. Tchoń and A. Matuszok, On Avoiding Singularities in Redundant Robot Kinematics, Robotica, 13 (1995) 599-606

[33] K. Tchoń and R. Muszynski, Singularities of Nonredundant Robot Kinematics, Int. J. Robotics Research, 16 (1997) 71-89

[34] K. Tchon, Singularities of the Euler Wrist, Mechanism and Machine Theory, 35 (2000) 505515

[35] D. K. Pai and M. C. Leu, Genericity and Singularities of Robot Manipulators, IEEE Trans. Robotics and Automation, 8 (1992) 545-559 
[36] J. Lerbet and K. Hao, Kinematics of Mechanisms to the Second Order-Application to the Closed Mechanisms, Acta Applicandae Mathematicae, 59 (1999) 1-19

[37] J. Lerbet, Singular Set of Four Screws: an Intrinsic Solution, Mechanism and Machine Theory, 38 (2003) 179-194

[38] P. Wenger, Classification of 3R Positioning Manipulators, ASME J. Mechanical Design, 120 (1998) 327-332

[39] M. Baili, P. Wenger and D. Chablat, Classification of One Family of 3R Positioning Manipulators, Proc. 11th Int. Conf. on Advanced Robotics, Coimbra, Portugal, (2003)

[40] M. Zein, P. Wenger and D. Chablat, An Exhaustive Study of the Workspace Topologies of all 3R Orthogonal Manipulators with Geometric Simplifications, Mechanism and Machine Theory, 41 (2006) 971-986

[41] A. Karger, Classification of Robot-Manipulators with only Singular Configurations, Mechanism and Machine Theory, 30 (1995) 727-736

[42] A. Karger, Classification of Serial Robot-Manipulators with Non-Removable Singularities, Trans. ASME J. Mechanical Design, 118 (1996) 202-208

[43] A. Karger, Singularity Analysis of Serial Robot-Manipulators, Trans. ASME J. Mechanical Design, 118 (1996) 520-525

[44] A. Ghosal and B. Ravani, A Differential-Geometric Analysis of Singularotoes of Point Trajectories of Serial and Parallel Manipulators, Trans. ASME J. Mechanical Design, 123 (2001) $80-89$

[45] P. S. Donelan, Generic Properties of Euclidean Kinematics, Acta Applicandae Mathematicae, 12 (1988) 265-286

[46] C. G. Gibson and P. E. Newstead, On the Geometry of the Planar 4-Bar Mechanism, Acta Applicandae Mathematicae, 7 (1986) 113-135

[47] C. G. Gibson and J. M. Selig, Movable Hinged Spherical Quadrilaterals I and II, Mechanism and Machine Theory, 23 (1988) 13-24

[48] C. G. Gibson and D Marsh, Concerning Cranks and Rockers, Mechanism and Machine Theory, 23 (1988) 355-360

[49] C. G. Gibson and J. M. Selig, On the Linkage Varieties of Watt 6-Bar Mechanisms I and II, Mechanism and Machine Theory, 24 (1989) 106-113

[50] C. G. Gibson and C. A. Hobbs, Simple Singularities of Space Curves, Math. Proc. Camb. Phil. Soc., 113 (1992) 297-310

[51] C. G. Gibson, Kinematic Singularities - A New Mathematical Tool, Proc. 3rd Int. Workshop on Advances in Robot Kinematics, Ferrara, Italy, (1992) 209-215.

[52] P. S. Donelan, C. G. Gibson and W. Hawes, Trajectory Singularities of General Planar Motions, Proc. Royal Soc. Edinburgh, 129A, (1999) 37-55 
[53] C. G. Gibson, W. Hawes and C. A. Hobbs, Local Pictures for General Two-Parameter Motions of the Plane, Advances in Robot Kinematics and Computational Geometry, Kluwer Academic Publishers, (1994) 49-58.

[54] C. G. Gibson and C. A. Hobbs, Local Models for General One-Parameter Motions of the Plane and Space, Proc. Royal Soc. Edinburgh, 125A (1995) 639-656

[55] C. G. Gibson and C. A. Hobbs, Singularity and Bifurcation for General Two-Dimensional Planar Motions, New Zealand J. Math., 25 (1996) 141-163

[56] C. G. Gibson, C. A.. Hobbs and W. L. Marar, On Versal Unfoldings of Singularities for General Two-Dimensional Spatial Motions, Acta Applicandae Mathematicae, 47 (1996) 221-242

[57] C. G. Gibson, D. Marsh and Y. Xiang, Singular Aspects of Generic Planar Motions with Two Degrees of Freedom, Int. J. Robotics Research, 17 (1998) 1068-1080

[58] R. Hines, D. Marsh and J. Duffy, Catastrophe Analysis of the Planar Two-Spring Mechanism, Int. J. Robotics Research, 17 (1998) 89-101

[59] J. P. Yin, D. Marsh and J. Duffy, Catastrophe Analysis of Planar Three-Spring Systems, in Proc. ASME Design Engineering Conf., Atlanta, (1998)

[60] M. Carricato, J. Duffy and V. Parenti-Castelli, Catastrophe Analysis of a Planar System with Flexural Pivots, Mechanism and Machine Theory 37 (2002) 693-716

[61] R. M. Murray, Z. Li and S. S. Shastry, A Mathematical Introduction to Robotic Manipulation, CRC Press, Boca Raton, 1994

[62] J. Selig, Geometrical Fundamentals of Robotics, Springer Verlag, New York, 2005

[63] J. Munkres, Topology, 2nd Edn., Prentice Hall, Upper Saddle River, NJ, 2000

[64] V. Guillemin and A. Pollack, Differential Topology, Prentice Hall, Englewood Cliffs, NJ, 1974

[65] J. M. Lee, Introduction to Smooth Manifolds, Springer, New York, 2002

[66] C. G. Gibson Singular Points of Smooth Mappings, Research Notes in Mathematics 25, Pitman, London, 1979

[67] V. I. Arnold, S. M. Gusein-Zade and A. N. Varchenko, Singularities of Differentiable Maps, Volume 1, Birkhauser, Cambridge MA, 1985

[68] J. Martinet, Singularities of Smooth Functions and Maps, LMS Lecture Note Series 58, Cambridge UP, 1982

[69] M. Golubitsky and V. Guillemin, Stable Mappings and Their Singularities, Springer Verlag, New York, 1973

[70] J. W. Burdick, On the Inverse Kinematics of Redundant Manipulators: Characterization of the Self-Motion Manifolds, Proc. IEEE Int. Conf. Robotics and Automation, (1989) 264-270 
[71] R. Brockett, Robotic Manipulators and the Product of Exponentials Formula, in Proc. Mathematical Theory of Networks and Systems, Beer-Sheva, Israel, ed. P Fuhrman, (1984) 120-129

[72] J. Denavit and R. S. Hartenberg, A Kinematic Notation for Lower Pair Mechanisms based on Matrices, ASME J. Applied Mechanics, 22 (1955) 215-221

[73] J. M. Hervé, Analyse Structurelle de Méchanismes par Group des Déplacement, Mechanism and Machine Theory, 13 (1978) 437-450

[74] C. G. Gibson and K. H. Hunt, Geometry of Screw Systems I \& II, Mechanism and Machine Theory, 25 (1990) 1-27

[75] P. S. Donelan and C. G. Gibson, First-Order Invariants of Euclidean Motions, Acta Applicandae Mathematicae 24 (1991) 233-251

[76] P. S. Donelan and C. G. Gibson, On the Hierarchy of Screw Systems, Acta Applicandae Mathematicae, 32 (1993) 267-296

[77] D. H. Gottlieb, Topology and the Robot Arm, Acta Applicandae Mathematicae, 11 (1988) $117-121$

[78] C. T. C. Wall, Regular Stratifications, in Dynamical Systems, Warwick, 1974, A. Manning (ed), Lecture Notes in Mathematics 468, Springer, New York (1975) 332-344

[79] H. Lipkin and E. Pohl, Enumeration of Singular Configurations for Robotic Manipulators, Trans. ASME J. Mechanical Design, 113 (1991) 272-279

[80] C. G. Gibson, K. Wirthmüller, A. A. du Plessis and E. J. N. Looijenga, Topological Stability of Smooth Mappings, Lecture Notes in Mathematics 552, Springer, Berlin, 1976

[81] J. W. Bruce, N. P. Kirk and A. A. du Plessis, Complete Transversals and the Classification of Singularities, Nonlinearity, 10 (1997) 253-275

[82] M. W. Cocke, P. S. Donelan and C. G. Gibson, Trajectory Singularities for a Class of Parallel Mechanisms, in Proc. 8th Intl. Workshop on Real and Complex Singularities, Luminy, France, 2004, eds. J.-P. Brasselet and M. A. S. Ruas, Birkhäuser, Basel (2006) 53-70

[83] J. L. Nevins and D. E. Whitney, Assembly Research, Automation, 16 (1980) 595-613

[84] D. E. Whitney and J. L. Nevins, What is the RCC and what can it do?, Robot Sensors, Tactile and Non-Vision, ed. A.Pugh, IFS Publications, (1986) 3-15

[85] M. W. Cocke, P. S. Donelan and C. G. Gibson, Instantaneous Singular Sets Associated to Spatial Motions, in Real and Complex Singularities, São Carlos, 1998, eds. F. Tari and J. W. Bruce, Res. Notes Math., 412 (Chapman and Hall/CRC Press, Boca Raton, 2000) 147-163 